\title{
The Fly Homologue of MFSD11 Is Possibly Linked to Nutrient Homeostasis and Has a Potential Role in Locomotion: A First Characterization of the Atypical Solute Carrier CG18549 in Drosophila Melanogaster
}

\author{
Mikaela M. Ceder ${ }^{1, *(\mathbb{D})}$, Frida A. Lindberg ${ }^{1}\left(\mathbb{D}\right.$, Emelie Perland ${ }^{1}\left(\mathbb{D}\right.$, Michael J. Williams ${ }^{2}$ and Robert Fredriksson $^{1}$ \\ 1 Molecular Neuropharmacology, Department of Pharmaceutical Biosciences, Uppsala University, \\ 75124 Uppsala, Sweden; frida.lindberg@farmbio.uu.se (F.A.L.); Emelie.perland@hotmail.com (E.P.); \\ robert.fredriksson@farmbio.uu.se (R.F.) \\ 2 Functional Pharmacology, Department of Neuroscience, Uppsala University, 75124 Uppsala, Sweden; \\ michael.williams@neuro.uu.se \\ * Correspondence: Mikaela.Ceder@neuro.uu.se; Tel.: +46-18-471-4511
}

Citation: Ceder, M.M.; Lindberg, F.A.; Perland, E.; Williams, M.J.; Fredriksson, R. The Fly Homologue of MFSD11 Is Possibly Linked to Nutrient Homeostasis and Has a Potential Role in Locomotion: A First Characterization of the Atypical Solute Carrier CG18549 in Drosophila Melanogaster. Insects 2021, 12, 1024. https://doi.org/10.3390/ insects12111024

Academic Editor: Ryusuke Niwa

Received: 13 October 2021

Accepted: 9 November 2021

Published: 13 November 2021

Publisher's Note: MDPI stays neutral with regard to jurisdictional claims in published maps and institutional affiliations.

Copyright: (C) 2021 by the authors Licensee MDPI, Basel, Switzerland. This article is an open access article distributed under the terms and conditions of the Creative Commons Attribution (CC BY) license (https:// creativecommons.org/licenses/by/ $4.0 /)$.
Simple Summary: The body is dependent on nutrients and ions to work normally. Within the hu-man body there is a group of proteins named transporters or solute carriers. These transporters are vital for the transport of nutrients such as glucose, amino acids, and fats, as well as ions such as sodium, calcium, and potassium. Despite being vital for normal physiology, as well as pathophysiology, a large number (approximately one third) of the transporters are orphans, where information about their expression and function is missing. Here, we aimed to begin to unravel the expression and function of one of these orphan transporters, MFSD11, by studying its orthologue in fruit flies (CG18549). We found that the fly orthologue is expressed in the brain of fruit flies and that it is possibly involved in metabolism and/or locomotion of the flies. The exact mechanism behind the observed behaviors is not fully understood, but our study provides new insights into the expression and function of CG18549. Clearly, these results, among others about the orphan transporters, provide a strong example as to why it is vital to fully characterize them and through that gain knowledge about the body during normal condition and disease.

\begin{abstract}
Cellular transport and function are dependent on substrate influx and efflux of various compounds. In humans, the largest superfamily of transporters is the SoLute Carriers (SLCs). Many transporters are orphans and little to nothing is known about their expression and/or function, yet they have been assigned to a cluster called atypical SLCs. One of these atypical SLCs is MFSD11. Here we present a first in-depth characterization of the MFSD11, CG18549. By gene expression and behavior analysis on ubiquitous and brain-specific knockdown flies. CG18549 knockdown flies were found to have altered adipokinetic hormone and adipokinteic hormone receptor expression as well as reduced vesicular monoamine transporter expression; to exhibit an altered locomotor behavior, and to have an altered reaction to stress stimuli. Furthermore, the gene expression of CG18549 in the brain was visualized and abundant expression in both the larvae and adult brain was observed, a result that is coherent with the FlyAtlas Anatomy microarray. The exact mechanism behind the observed behaviors is not fully understood, but this study provides new insights into the expression and function of CG18549. Clearly, these results provide a strong example as to why it is vital to fully characterize orphan transporters and through that gain knowledge about the body during normal condition and disease.
\end{abstract}

Keywords: solute carrier; MFS; MFSD11; CG18549; drosophila melanogaster; locomotion 


\section{Introduction}

Transporters are essential for substrate absorption, distribution, metabolism, and elimination, and they are evolutionary conserved in prokaryotes and eukaryotes [1-4]. The largest protein family clan of functionally related transporter and transporter-like proteins across several phyla, with over 1,000,000 sequences members divided into 87 recognized families [5], is the Major Facilitator Superfamily (MFS) [6-9]. In the late eighties, all members of the MFS were presumed to facilitate transport of various sugars [10], but today they are known as a diverse transporter family $[7,11]$. In humans, the largest superfamily of transporters is the SoLute Carrier (SLC) proteins, and approximately one third of the human SLCs populates the MFS Pfam clan ([12], slc.bioparadigms.org, accessed on 16 March 2021). The main function of SLCs is to transport solutes across the plasma and organelle membranes and provide cells with, e.g., carbohydrates, amino acids, lipids, and ions [2,3]. The function of SLCs can roughly be divided into four categories: cotransporters (symporters), exchangers (antiporters), facilitators (uniporters) and orphans (slc.bioparadigms.org, accessed on 16 March 2021) [2,3]. However, lately, two subfamilies of the SLCs, the Proton-assisted Amino acid Transporter (PAT or SLC36) and the System A and System N sodium-coupled neutral amino acid transporter family (SNATs, SLC38), have gained attention for their involvement in not only transport but also nutrient sensing. Moreover, both families have been linked to mammalian target of rapamycin complex 1 (mTORC1) activation and function [13]. Hence, it is not surprising that one fourth of all SLCs are associated with diseases [14]. However, despite the associations with, e.g., neurological diseases and metabolic disorders, only a few SLCs are used as drug targets [15]. Also, one third of the SLCs are still categorized as orphans and, unfortunately, the SLCs remain understudied compared to other integral membrane proteins [12,14].

In 2017, Perland and colleagues suggested that there are more transporter and transporterrelated proteins that ought to be classified as SLCs, and they named this group "Atypical SoLute Carriers" [12,16]. A couple of years later, this was found to be true when the SLC superfamily grew from 52 subfamilies with approximately 393 members to 65 subfamilies with 430 members (no pseudo genes counted) (slc.bioparadigms.org, accessed on 16 March 2021). A large proportion of these atypical SLCs have similarities with the MFS and are therefore called Major Facilitator Superfamily domain (MFSD) proteins (MFSD1-MFSD14). Among the 65 subfamilies of SLCs, six subfamilies contain one or more MFSD protein (slc.bioparadigms.org, accessed on 16 March 2021).

Herein, we have studied the orphan MFSD protein MFSD11 (Major Facilitator Superfamily containing Domain 11). MFSD11 is evolutionary conserved, with orthologs found in simple species as C. elegans, C. intestinalis and D. melanogaster, as well as in more complex species as humans, apes, and rodents [17]. Little is known about its function, but genomewide association studies predict that MFSD11 could be involved in Retinal arteriolar caliber (study accession: GCST002071) [18] and total paired-helical-filament (PHF) tau (study accession: GCST010340) [19]. At present, there are only four publications focusing directly on MFSD11: a characterization study focusing on the expression pattern of MFSD11 in mouse [17]; a broad study about putative SLCs and their connections to sugar concentrations [20]; a study focusing in mutations associated with trastuzumab-resistance [21] and a genomic study where it was suggested to be a novel intellectual disability (ID) candidate [22]. The gene expression of $M f s d 11$ in mice is widespread, with expression in both the peripheral and the central nervous system (CNS). The MFSD11 protein is abundantly found in the mouse brain, both in embryonal and adult tissue and it is expressed in neurons positive for both anti-glutaminase and vesicular inhibitory amino acid transporter (VIAAT) [17] Furthermore, databases as GTEX reports that human MFSD11 is moderately expressed across tissues and its lowest expression is in brain, pancreas and whole blood (Data Source: GTEx Analysis Release V8 (gtexportal.org (accessed on 16 March 2021), dbGaP Accession phs000424.v8.p2, 21 June 2021). There have been speculations that MFSD11 could be involved in metabolism or at least that it is a gene that reacts highly to the nutritional status 
of the body, i.e., the gene expression of $M f s d 11$ has been found to be affected by sugar concentrations and starvation in both in vitro and in vivo models $[17,20]$.

The aim of this project was to characterize MFSD11 with focus on both expression and function by studying the orthologous gene in D. melanogaster, CG18549. Expression of the gene was investigated using quantitative polymerase chain reactions (qPCRs) and visualized using an enhancer-trap line that drives green fluorescent protein in CG18549 positive cells. Starvation and behaviors linked to locomotion, age and stress were studied with the Drosophila Activity Monitor System (DAMS) on ubiquitous and brain specific knockdown flies. We found that (I) CG18549 expression is regulated by nutrient availability, (II) CG18549 knockdown flies exhibit an altered locomotor behavior and (III) have an altered stress response. Unfortunately, the exact mechanism behind the observed behaviors is not fully understood. Taken together, the data suggest that CG18549 has a potential role in locomotion behavior and possibly in metabolism, either by directly or indirectly affecting genes linked to these processes.

\section{Materials and Methods}

\subsection{Prediction of Secondary and Tertiary Structure}

Models of the human MFSD11 and D. melanogaster CG18549 were predicted using Phyre2 [23]. The MFSD11 (Uniprot ID O43934) and the CG18549 (Flybase ID FBpp0082101) structures were modeled against the template with the highest confidence, sequence coverage and identity. The protein unc-93 homolog b1 from mus musculus (PDB id c7c77B) [24] were used for prediction of MFSD11 and CG18549 secondary and tertiary structures. MFSD11 was modeled with $11 \%$ identity, 100\% confidence and $94 \%$ amino acid coverage (423 out of 449 residues). CG18549 was modeled with $12 \%$ identity, $100 \%$ confidence and $97 \%$ amino acid coverage (424 out of 436 residues). Phyre2 provided backbone images of both the secondary and tertiary predictions. The gradient of the tertiary structures displays helices from N-terminal (dark blue) to C-terminal (red), and unconnected loops in the predictions are parts of the structure that have uncertainties within the model; therefore, they cannot be fully modeled.

\subsection{Fly Stocks and Maintenance}

The $\left.{ }^{*}{ }^{*}\right] ; P\{w[+m C]=G A L 4-e l a v . L\} 3, P\{G a w B\}$ elavC155 $w^{*} ; P\{F R T$ (whs) $\}$ G13P $\{$ tubPGAL80\}LL2, y[1] sc[*] v[1]; P\{y[+t7.7] v[+t1.8]=TRiP.HMS01385\}attP2 (CG18549 RNAi line 1, Stock no. 39341, tested for functionality with da-GAL4), w[1118] and Pin/Pin; UASmCD8-GFP (derived from yw;Pin/Cyo, UAS-mCD8-GFP, stock no. 5130) received from the Bloomington Stock Center, the $y\left[{ }^{*}\right] \mathrm{w}\left[{ }^{*}\right] ; \mathrm{P}\{\mathrm{w}[+\mathrm{mW} \cdot \mathrm{hs}]=$ GawB $\}$ CG18549[NP5325]/TM6, $\mathrm{P}\{\mathrm{w}[-]=\mathrm{UAS}-\mathrm{lacZ}$.UW23-1\}UW23-1 was received from Kyoto Drosophila Stock Center, and P\{KK102196\}VIE-260B (CG18549 RNAi line 2, Stock no. v107272, tested for functionality with da-GAL4) was received from Vienna Drosophila RNAi Center (VDRC). Da-Gal4 flies were a generous gift from Professor D. Nässel and CSORC flies were a generous gift from Associate Professor and Dr. M.J. Williams (cross of CantonS and OregonR-C, [25]). All flies were crossed into the same $\mathrm{w}^{1118}$ background. In all assays, the GAL4 drivers and UAS transgenic flies were crossed to $\mathrm{w}^{1118}$ flies and their F1 progeny were used as controls.

All flies, unless otherwise stated, were maintained on enriched Jazz mix standard fly food (Fisher Scientific, Stockholm, Sweden) supplemented with yeast extract (VWR). Fly stocks were maintained at $25^{\circ} \mathrm{C}$ in an incubator at $60 \%$ humidity on a $12 \mathrm{~h}: 12 \mathrm{~h}$ light:dark cycle. Flies crossed to GAL4 drivers and controls were raised at $18^{\circ} \mathrm{C}$ until adults emerged. Once collected, adults were raised at either 25 or $29^{\circ} \mathrm{C}$, depending on the driver line, for the appropriate times.

\subsection{Gene Expression Analysis Using Fluorescent Protein}

The gene expression of CG18549 in the brain of third instar larvae and adult flies were studied by using F1 progenies from the CG18549-GAL4 and Pin/Pin-UAS-GFP cross, and the progenies from each line separate were used as background controls. Larvae were col- 
lected and the brain and ventral nerve cord were dissected in 1xPBS-tx on a silicone coated petri dish. The tissues were rinsed three times to remove membranes and excessive artifacts, before being mounted on Superfrost Plus slides in VectaShield (H-1000, Vector Laboratories, Stockholm, Sweden). Images were captured using fluorescent Axioplan 2 imaging microscope. Adult flies were anesthetized with $\mathrm{CO}_{2}$ before decapitation and fixation in $8 \%$ formaldehyde for one hour, followed by washing in 1xPBS $15 \mathrm{~min}$ three times. The brains were retrieved and either mounted on Superfrost Plus slides in VectaShield (H-1000, Vector Laboratories) and imaged using fluorescent Axioplan 2 imaging microscope or mounted on 1\% agarose (Conda) in petri dishes for imaging in a Zeiss LSM710 2 Photon microscope with the Zen black software. Experimental and control images were captured with the same exposure time.

\subsection{Diet and Starvation mRNA Panels}

\subsubsection{Diet Panel}

This method was modified from $[20,26]$. All CSORC flies ( $n=5$, with 20 male flies in each replicate) were five days old and fed for five days, and then euthanized by freezing at $-80{ }^{\circ} \mathrm{C}$. The flies were decapitated, and total RNA was extracted from the heads. Data from fly body was used with permission and modified from [20].

The following sugar and yeast (protein) ratios were used when preparing the diets: 10:10 g/dL (control), 2.5:2.5 g/dL, 40:40 g/dL, 2.5:40 g/dL, 10:40 g/dL, 40:2.5 g/dL and 40:10 g/dL.

\subsubsection{Starvation Panel}

This method was modified from [20]. Briefly, five- to seven-days-old CSORC males ( $n=5-7$, with 20 flies on each replicate) were collected at $0(n=7), 3(n=7), 6(n=5)$, $9(n=5), 12(n=6)$ and $24(n=6) h$ of starvation before euthanized by freezing at $-80^{\circ} \mathrm{C}$. The flies were decapitated, and total RNA was extracted from the heads. Data from fly bodies were used with permission and modified from [20].

\subsection{RNA Extraction, cDNA Synthesis, Primer Design and $q P C R$}

RNA extraction, cDNA synthesis, primer design and qPCR were performed as described in $[20,27]$.

RNA extraction: In total ten flies per replicate were collected for RNA extraction of whole fly, while 20 flies per replicate were collected for RNA extraction of heads. The RNA pellet was dissolved in $20 \mu \mathrm{L}$ of DEPC water, and the concentration was measured in a ND-1000 spectrophotometer (NanoDrop Technologies, Wilmington, DC, USA).

cDNA synthesis: Two $\mu \mathrm{g}$ of cDNA was synthesized with High-Capacity RNA-tocDNA kit (Applied Biosystems, Waltham, MA, USA) according to the manufacturer's instructions. The samples were diluted to $10 \mathrm{ng} / \mu \mathrm{L}$ RNA in sterile water.

Primer design: Primers were designed with Beacon Design 8 (Premier Biosoft, Palo Alto, CA, USA). Primers are summarized in Table 1, where reference genes are in red and target genes are in black.

qPCR: Ct Values were obtained using the CFX Maestro (BioRad, Stockholm, Sweden) and primer efficiencies were calculated via LinRegPCR software. The delta $\mathrm{Ct}$ methods for either one (Actin42A) or several reference genes (Actin42A, Rpl11, Rp49 and Rpl13A) (according to [28]) were used to normalize and calculate the relative mRNA expression presented as fold differences. The log 2 fold change $( \pm 95 \% \mathrm{CI})$ was plotted, and the gene expressions were compared to the Driver control. Expression differences were analyzed with Kruskal-Wallis with Dunn's comparison or Mann-Whitney (specified in figure legends) using GraphPad Prism, version 5. 
Table 1. Display the name and the forward and reverse sequence of primers. Housekeeping genes are marked in red.

\begin{tabular}{ccc}
\hline Primer & Forward & Reverse \\
\hline Actin42A & acaacacttccgctcct & gaacacaatatggttgcttatgc \\
\hline$A k h$ & tagtgctgtgttaattac & tcattctgagttctatg \\
\hline$A k h R$ & aggagcgacttgatgag & tccgtagcagtagatgaa \\
\hline CG18549 & tgttcgtcttacggcattcc & gtgtagccctcacccttgaa \\
\hline DAT & gcttcaaaccataagttctaa & tcggacttgatattatctacaa \\
\hline Dilp2 & ctgcagtgaaaagctcaacga & tcattctgagttctatg \\
\hline Dilp3 & tgaacgaactatcactcaacagtct & agagaacttggacccotgaa \\
\hline Dilp5 & gaggcaccttgggcctattc & catgtggtgagattcggagct \\
\hline Dilp6 & gtccaaagtcctgctagtcct & tctgttcgtattccgtgggtg \\
\hline InR & caagccgttcgtccatc & tcattccaaagtcaggaa \\
\hline Pale & tcaagaaatcctacagtat & cacaatgcaatcttccag \\
\hline Rp49 & cacaccaatcttacaaatgtgtga & aatccggcttgcacatg \\
\hline Rpl11 & ccatcggtatctatggtctgga & catcgtatttctggaacc \\
\hline Vmat & gtgaccttcgggacgatag & actagagcgggaaaaccagc \\
\hline
\end{tabular}

\subsection{Offspring Counting}

F1 progenies from $d a-G A L 4>w^{1118}$ (Driver ctrl), $w^{1118}>$ CG18549 RNAi (RNAi ctrl) and $d a-G A L 4>$ CG18549 RNAi (CG18549 knockdown) were collected and examined. Both CG18549 RNAi lines were used, and the counting was performed on progenies from three crosses of each genotype. The mean $( \pm 95 \% \mathrm{CI})$ are plotted for male and female progenies separated as well as merged for each RNA line. Differences were analyzed using KruskalWallis with Dunn's comparison. Progenies were investigated for developmental flaws using a Leica M125 microscope with a ProgRes C14 plus camera (Jenoptik) and the ProgRes CapturePro 2.8 Jenoptik Optical system.

\subsection{RNA Sequencing}

Sequencing libraries were prepared from $1 \mu \mathrm{g}$ total RNA using the TruSeq stranded mRNA library preparation kit (Cat\# 20020594/5, Illumina, Uppsala, Sweden) including polyA selection. The library preparation was performed according to the manufacturers' protocol. Sequencing was performed on one lane on the NovaSeq SP flowcell, paired-end $150 \mathrm{bp}$ read length, using v1 chemistry on a NovaSeq 6000 system (Illumina).

The sample files are stored in the SRA repertoire and can be found under PRJNA689052. The reads were mapped against the D. melanogaster genome assembly 6.09 using STAR mapper (25). Mapping was undertaken against a genome index generated with the FlyBase GTF annotating file for the 6.09 genome assembly to direct mapping toward annotated genes. A total of 326,643,821 transcripts were mapped uniquely and used in subsequent analysis. The assembled transcripts were used in DESeq2 (26) to obtain a final transcriptome assembly and to calculate the relative and differential expression, summarized in Supplementary Data sheet 1 . Genes were considered to be differentially expressed if they had a $\log 2$ fold change $>1$ or $<-1$ in the DESeq2 analysis. An ANOVA with FDR correction was used to identify expression differences between all three sample groups, and Wald test with Benjamini and Hochberg multiple correction method was used as post hoc test. The $\log 2$ fold changed genes from the qPCR runs were compared with the RNA sequencing $\log 2$ fold change and summarized in Table 2. 
Table 2. RNA sequencing log2 fold change as a validation of qPCRs run on the Driver control (da-GAL4 > w1118), the RNAi control (w1118 > CG18549 RNAi line 1) and the CG18549 knockdown (da-GAL4 > CG18549 RNAi line 1) flies. The table summarize the gene, flybase.org (accessed on 16 March 2021) identity (FB ID) and the log2 fold change and Wald test $p$-values.

\begin{tabular}{|c|c|c|c|c|c|c|c|}
\hline \multirow{3}{*}{ Gene } & \multirow{3}{*}{ FB ID } & \multicolumn{6}{|c|}{ Wald Test } \\
\hline & & \multicolumn{2}{|c|}{ Driver vs. RNAi } & \multicolumn{2}{|c|}{ Driver vs. Knockdown } & \multicolumn{2}{|c|}{ RNAi vs. Knockdown } \\
\hline & & $\begin{array}{l}\text { Log2 Fold } \\
\text { Change }\end{array}$ & $p$-Value & $\begin{array}{l}\text { Log2 Fold } \\
\text { Change }\end{array}$ & $p$-Value & $\begin{array}{l}\text { Log2 Fold } \\
\text { Change }\end{array}$ & $p$-Value \\
\hline CG18549 & FBgn0038053 & 0.27 & 0.57 & -0.47 & 0.32 & -0.74 & 0.15 \\
\hline Akh & FBgn0004552 & 0.35 & 0.27 & 0.66 & 0.04 & 0.31 & 0.36 \\
\hline$A k h R$ & FBgn0025595 & -0.19 & 0.36 & -0.34 & 0.09 & -0.15 & 0.49 \\
\hline Ilp2 & FBgn0036046 & 0.70 & 0.09 & 1.16 & 0.004 & 0.45 & 0.30 \\
\hline Ilp3 & FBgn0044050 & 0.33 & 0.34 & 0.65 & 0.06 & 0.32 & 0.38 \\
\hline Ilp5 & FBgn0044048 & 0.09 & 0.86 & 1.05 & 0.04 & 0.96 & 0.07 \\
\hline Ilp6 & FBgn0044047 & -0.04 & 0.76 & -0.15 & 0.31 & -0.10 & 0.50 \\
\hline $\operatorname{In} R$ & FBgn0283499 & $\mathrm{N} / \mathrm{A}$ & $\mathrm{N} / \mathrm{A}$ & $\mathrm{N} / \mathrm{A}$ & $\mathrm{N} / \mathrm{A}$ & $\mathrm{N} / \mathrm{A}$ & $\mathrm{N} / \mathrm{A}$ \\
\hline Dat & FBgn0034136 & -0.29 & 0.27 & -0.27 & 0.30 & 0.02 & 0.96 \\
\hline Dop1R1 & FBgn0011582 & -0.56 & 0.05 & -0.56 & 0.05 & -0.01 & 0.99 \\
\hline Dop1R2 & FBgn0266137 & 0.13 & 0.66 & -0.31 & 0.29 & -0.44 & 0.16 \\
\hline Dop $2 R$ & FBgn0053517 & -0.08 & 0.90 & -1.25 & 0.03 & -1.17 & 0.06 \\
\hline Ple & FBgn0005626 & -0.45 & 0.17 & -0.93 & 0.005 & -0.48 & 0.18 \\
\hline Vmat & FBgn0260964 & -0.14 & 0.60 & -0.74 & 0.007 & -0.60 & 0.05 \\
\hline
\end{tabular}

\subsection{Drosophila Activity Monitor System}

Starvation resistance assay and locomotion, as well as age-dependent locomotion, were performed as described in $[29,30]$. Details about the stress assay and the filming of start and stop movement are described below. All experiments were performed on five- to seven-days-old male flies except for the age-dependent locomotion where the flies were aged to 13 days and to 21 days. A minimum of eight (range $n=8-30)$ individual flies were used for each setup; the exact number and driver lines used for the different experiments are described below under the specific subheading and/or specified in figure legends. All data were retrieved via Trikinetic software. Parameters of flies that deceased during the locomotion assays were removed as outliers. Other outliers were removed using GRUBBS outlier test, with $\alpha=0.05$. GraphPad Prism v.5 software was used to generate graphs and perform statistical analyses. Total beam breaks, activity (beam breaks) per hour and data points from filming are presented as scatter plots (mean $\pm 95 \% \mathrm{CI}$ ) and total activity was also plotted over time in a point-connected line graph (mean). Starvation resistance was presented as a scatter plot (mean $\pm 95 \% \mathrm{CI}$ ) to give an indication of total hours survived, also a survival proportion plot and a point-connection line graph were constructed to illustrate the survival over time and locomotion. Differences were calculated using Kruskal-Wallis with Dunn's comparison and/or Mann-Whitney as a post hoc test to calculate exact p-values (specified in figure legends).

Starvation resistance: In short, adult male flies from Driver control, RNAi control and CG18549 knockdown ( $d a-G A L 4$ driver, $\mathrm{n}=30$ ) were transferred to $5 \mathrm{~mm}$ glass tubes, prepared with $1 \%$ agarose, and contained in the DAMS until the last beam break. The last beam crossing per fly was defined as the timepoint of death.

Locomotion: F1 progenies from Driver control, RNAi control and CG18549 knockdown (da-GAL4 driver $\mathrm{n}=30$, elav-GAL4 driver $\mathrm{n}=10$, elav-GAL4:GAL80 driver (onset 1-day post-eclosion) $\mathrm{n}=10$ ) were transferred to $5 \mathrm{~mm}$ glass tubes, prepared with enriched Jazz mix standard food, contained in the DAMS for 24 to $72 \mathrm{~h}$ to record the activity level 
(locomotion, beam breaks). Age-dependent locomotion was performed on 13 (Driver ctrl $\mathrm{n}=9$, RNAi ctrl $\mathrm{n}=12$, CG18549 knockdown $\mathrm{n}=24)$ and $21(\mathrm{n}=30)$ days old male Driver control (elav-GAL4 driver), RNAi control and CG18549 knockdown flies with the same setup as the other locomotion assays.

Stress: Stress assay was performed on Driver control (elav-GAL4 driver), RNAi control and CG18549 knockdown flies $(n=10)$ for two days $(\sim 48 \mathrm{~h})$. Stress was induced by exposing the flies to bright light (LED, 1500 lumens) for $10 \mathrm{~min}$ three times (9.00, 12.00 and $15.00 / 16.00)$ per day. The total activity over $48 \mathrm{~h}$ was plotted in a line graph and the arrows indicate the timepoints where the flies were exposed to light. The total beam breaks for each day were calculated as well as the total beam break one hour after each stressor.

Filming: A 24 well tissue culture plate was coated with 1\% agarose (conda). Adult flies (elav-GAL4:GAL80 driver, $\mathrm{n}=8$ per group) were transferred to the plate one fly to each well. The flies were left for $5 \mathrm{~min}$ before filming started. The film was recorded by a Panasonic HC-V700 Full HD video camera. Flies were filmed for $20 \mathrm{~min}$ and the time of activity was manually measured for each fly using a stopwatch.

\subsection{Effect Size Calculations}

Effect sizes were calculated using mean differences $\left(\right.$ Mean $_{\text {target }}-$ Mean $\left._{\text {control }}\right)$ via www.estimationstats.com (accessed on 16 March 2021) according to [31]. Briefly, the mean differences for comparisons are plotted in a Cumming estimation plot. The raw data is plotted on the upper axes; each mean difference is plotted on the lower axes as a bootstrap sampling distribution. Mean differences are depicted as dots; $95 \%$ confidence intervals (CI) are indicated by the ends of the vertical error bars, and is bias-corrected and accelerated. Permutation tests are performed with 5000 reshuffles of the control and test labels, and the $p$-values equals the likelihoods of observing the effect sizes.

\section{Results}

\subsection{MFSD11 and CG18549 Have Similar Secondary and Tertiary Structure}

MFSD11 is known to be evolutionary conserved and is found in several other species. Phylogenetic analyses and amino acid alignments have been performed previously, revealing that the closest homologue to MFSD11 is CG18549 and that they have been reported to have $41.5 \%$ identical amino acid residues when performing a global pair-wise alignment $[17,27]$. Herein, we wanted to study the secondary and tertiary structures to look at similarities in the 2D and 3D structures, which could tell us more about their relationship and how well the results can be translational. The secondary and tertiary structure were predicted using Phyre2 [23]. The homology modeling predicted that MFSD11 and CG18549 have a similar secondary structure as other MFS proteins with 12 transmembrane helices [6], Figure 1A,B. Both proteins were also found to have the MFS loop between the sixth and seventh transmembrane helices, something that have been reported previously for atypical SLCs of MFS type [32,33], Figure 1A,B. Similar to one of their closest relative proteins, UNC93A [32], both MFSD11 and CG18549 were modeled with short N-terminus and C-terminus. Both MFSD11 and CG18549 were modeled against the crystal structure of UNC93B1, and are therefore predicted to have large similarities in the global protein folding patterns, Figure 1C,D. Both predications were modeled with high confidence and amino acid coverage, which indicates that MFSD11 and CG18549 most likely adopts to the overall fold illustrated in Figure 1. 
A

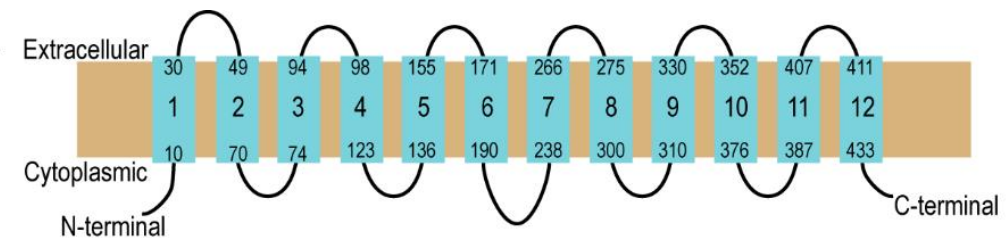

B
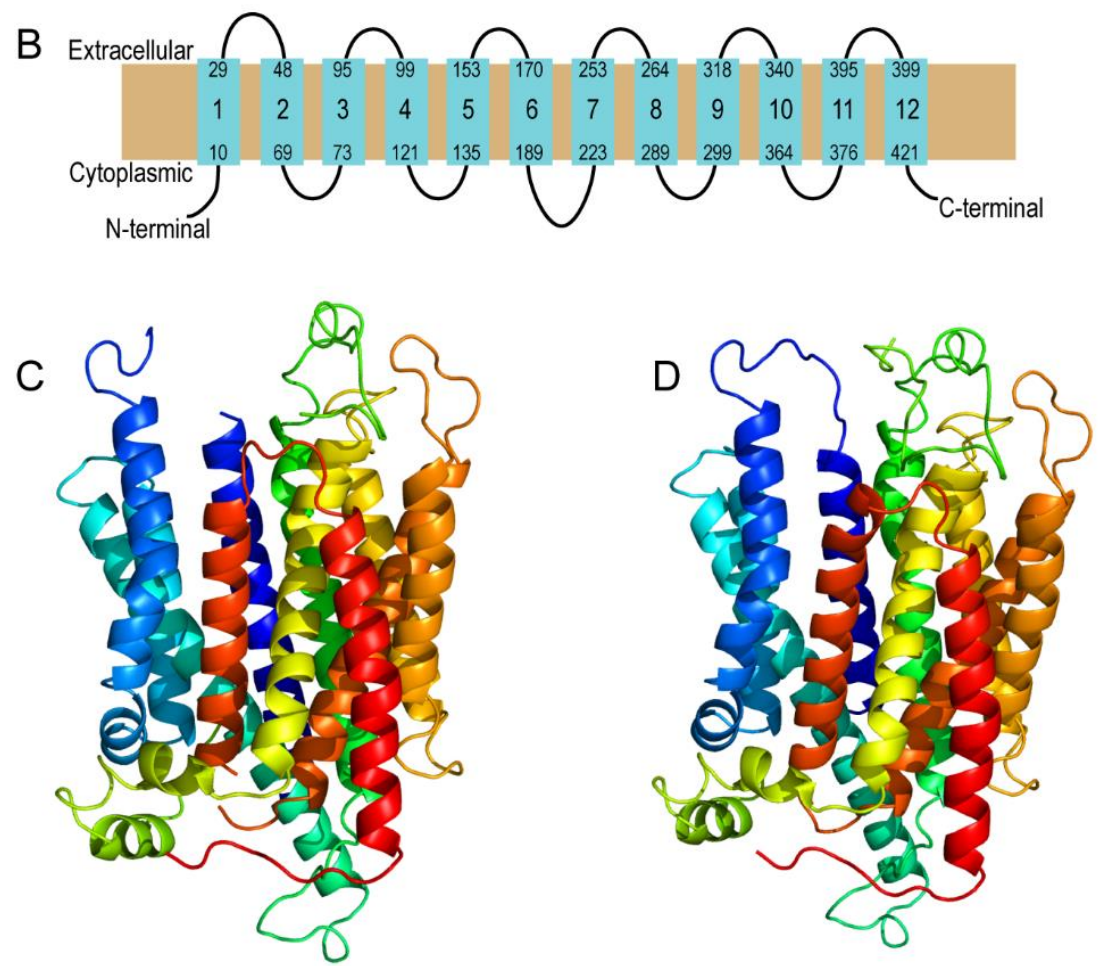

Figure 1. Prediction of secondary and tertiary structure of human MFSD11 and D. melanogaster CG18549. Homology modeling and predictions were performed using Phyre2 [23]. Both proteins were modeled against unc-93 homolog b1 from Mus musculus (PDB id c7c77B) [24]. (A) Secondary structure of human MFSD11. It was modeled with $11 \%$ identity, $100 \%$ confidence and $94 \%$ amino acid coverage. (B) Secondary structure of D. melanogaster CG18549. It was modeled with 12\% identity, $100 \%$ confidence and $97 \%$ amino acid coverage. (C) Tertiary structure of human MFSD11 and (D) D. melanogaster CG18549. The gradient of the tertiary structures displays helices from N-terminal (dark blue) to C-terminal (red). Unconnected loops in the predictions are parts of the 3D structure that are modeled with uncertainties and, therefore, they cannot be fully modeled.

\subsection{The CG18549 Gene Is Abundantly Expressed in the Larvae and Adult Brain}

MFSD11 has previously been reported to be an abundantly expressed gene and protein in mice, and immunostaining was reported to be present in embryos and adult mice [17] with high expression observed in the central nervous system (CNS). Furthermore, through the FlyAtlas [34] a majority of the genes in D. melanogaster have been mapped via microarray data and RNA sequencing data [35], and the data is summarized on flybase.org (accessed on 16 March 2021). According to these expression data sets, CG18549 is abundantly expressed, with moderate expression in the brain and CNS of larvae and adult flies, and high expressions in the Malpighian tubules, fat body and salivary glands [34]. Interestingly, it is also reported by Fly-FISH (a database of Drosophila embryo and larvae mRNA localization patterns) that CG18549 is expressed during different embryo stages [36,37]. However, these data are based on microarray and/or in situ and the expression has so far not been imaged in adult flies. Herein, we focused on the brain expression of CG18549 in adult flies to be able to compare with previous findings of CG18549 and Mfsd11, which have been based on brain tissue. To be able to visualize the CG18549 gene expression in brain cells, we crossed the enhancer-trap line CG18549-GAL4 and a UAS-GFP line, which results in progenies 
that will have CG18549 positive cells labeled with green fluorescent protein (GFP). Brains were dissected from five- to seven-days-old male flies, and images were captured with a fluorescent microscope, Figure 2. CG18549-GFP signals were visible in the brain and ventral nerve cord of third instar larvae $(\mathrm{n}=2)$, Figure $2 \mathrm{~A}, \mathrm{~B}$, but not in the separate lines, Figure 2C,D. GFP was also observed in the adult brain, Figure 2E, and no specific signals were visual in the controls, Figure 2F,G. Unfortunately, a fluorescent Axioplan 2 imaging microscope was not able to image individual cells; therefore, three additional brains were dissected and imaged with a Z-stack using a Zeiss LSM710 2 Photon microscope. The images, Figure 2H-J, revealed that CG18549 was expressed in different regions of the adult brain and both larger and smaller cells were GFP-positive, and projections were found to be labelled with GFP. However, no double staining was performed and hence, the exact cell type cannot be determined.
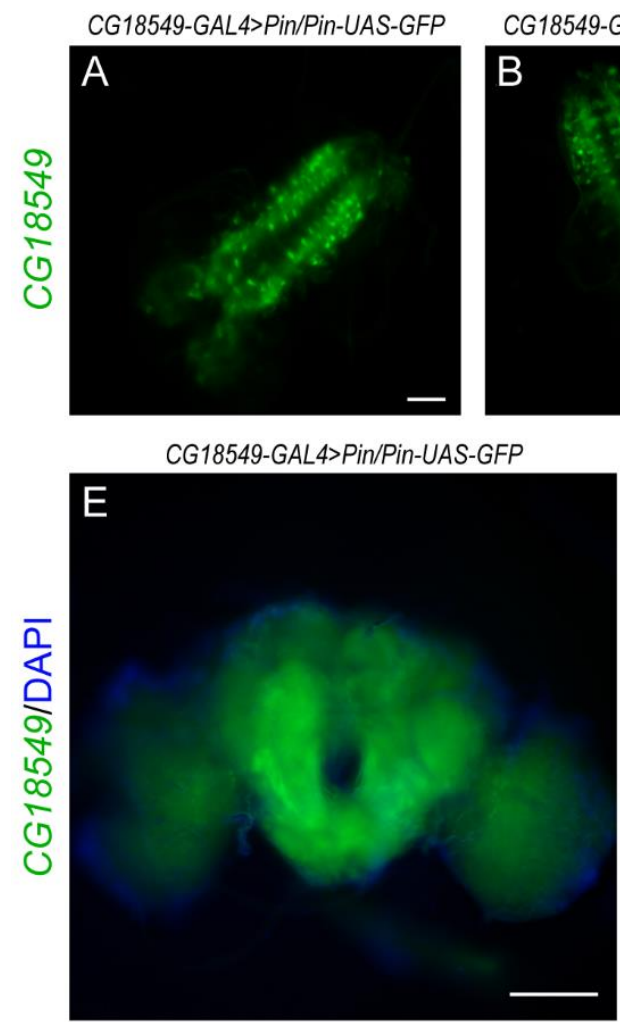

CG18549-GAL4>Pin/Pin-UAS-GFP

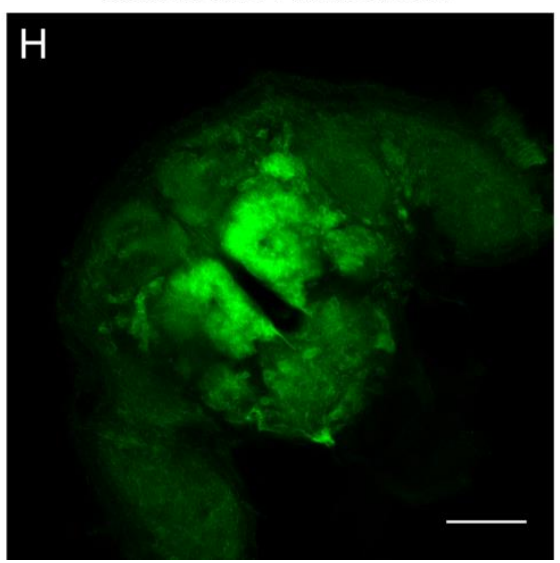

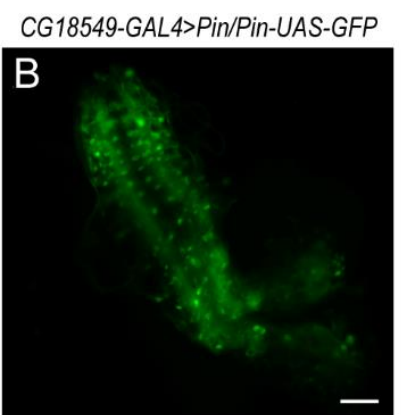

CG18549-GAL4

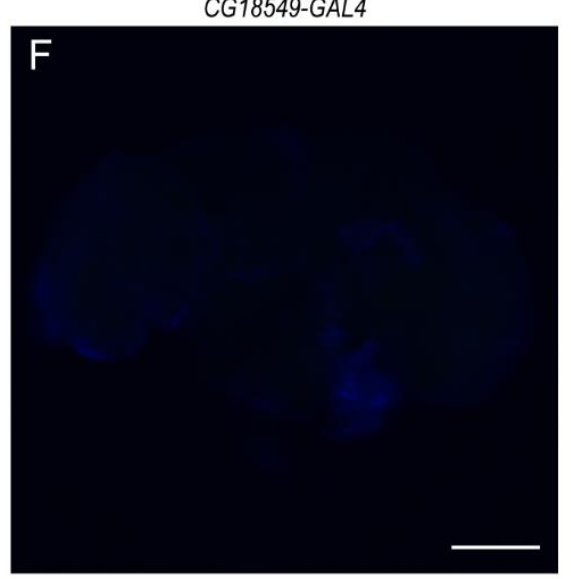

CG18549-GAL4>Pin/Pin-UAS-GFP

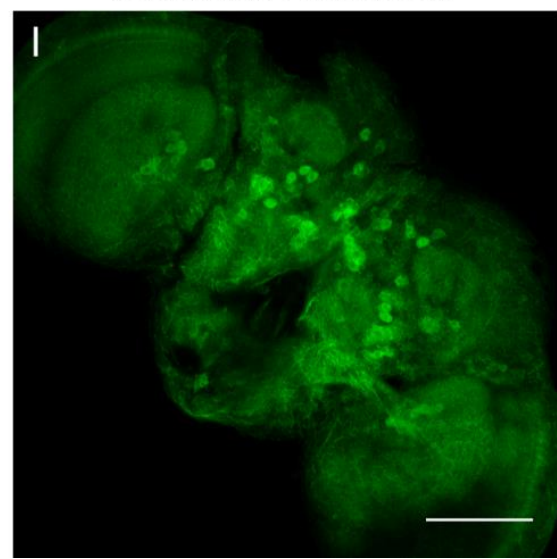

CG18549-GAL4
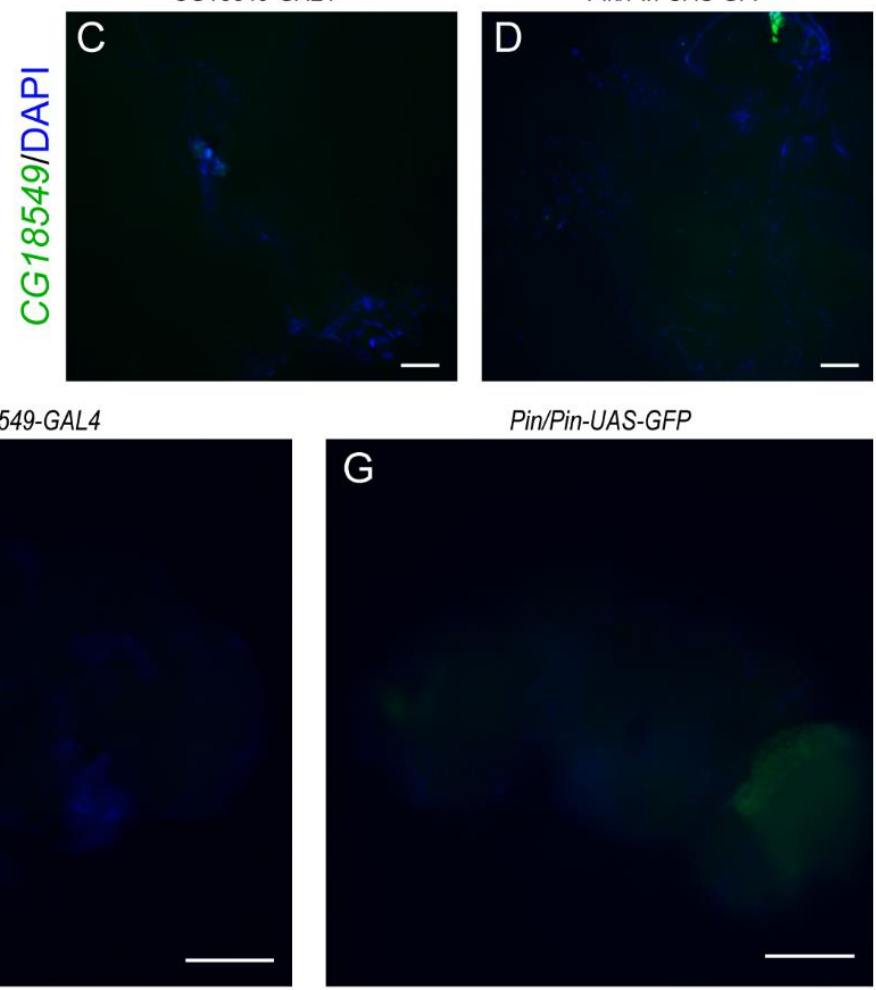

CG18549-GAL4>Pin/Pin-UAS-GFP

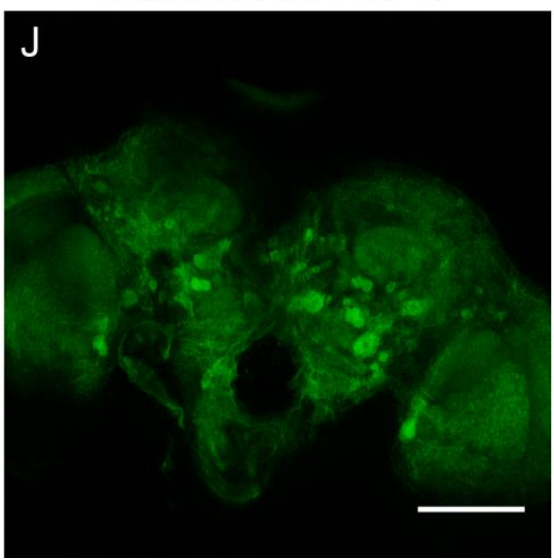

Figure 2. CG18549 gene expression images of the third instar larvae CNS and the adult brain. (A-D) Dissected brains and ventral nerve cord from third instar larvae, CG18549 expression was visualized with green fluorescence and blue (DAPI) marks the nucleus, scale bar $20 \mu \mathrm{m}$. (A,B) CG18549-GAL4 > Pin/Pin-UAS-GFP, (C) CG18549-GAL4 and (D) Pin/Pin-UAS-GFP larvae. 
(E-G) Dissected brains from seven-days-old adult flies, CG18549 expression was visualized with green fluorescence and blue (DAPI) marks the nucleus, scale bar $100 \mu \mathrm{m}$. (E) CG18549-GAL4 > Pin/Pin-UAS-GFP, (F) CG18549-GAL4 and (G) Pin/PinUAS-GFP adult flies. (H-J) 2-photon, high resolution images of CG18549-GAL4 > Pin/Pin-UAS-GFP (n = 3) seven-days-old flies. CG18549 expression was visualized with green fluorescence and blue (DAPI) marks the nucleus, scale bar $100 \mu \mathrm{m}$. Brains were imaged with Z-stacks and the pictures were merged displaying the average intensity. (H) Picture include $60(1 \mu \mathrm{m})$ slides, (I) picture include $68(1 \mu \mathrm{m})$ slides, $(\mathbf{J})$ Picture include $29(1 \mu \mathrm{m})$ slides.

\subsection{Functional Validation of Two RNAi Lines}

So far, limited data has been gathered about MFSD11 and its orthologues; to date, no functional characterization has been performed to understand its physiological role. To be able to perform functional studies on CG18549 and to study its physiological role, one commonly used technique within the research field of transporters is to manipulate the gene expression level. In D. melanogaster, there are sophisticated tools and fly lines available to perform knockdown studies on the desired gene. Herein, we decided to initiate the first in-depth functional characterization of CG18549 by performing RNAi knockdown. Two different CG18549 RNAi lines were commercially available and used here. They were tested for functionality using the ubiquitous da-GAL4 driver and measure the relative mRNA expression in three crosses: Driver control (da-GAL4 > w1118), RNAi control (w1118 > CG18549 RNAi) and CG18549 knockdown (da-GAL4 > CG18549 RNAi). The effect sizes of both lines were estimated by calculating the unpaired mean differences between the $\log 2$ fold values (Driver control vs. RNAi control, Driver control vs. CG18549 knockdown, RNAi control vs. CG18549 knockdown) and two-sided permutation $t$-tests were performed; the log2 fold differences and mean differences are illustrated in Figure $3 \mathrm{~A}$, and the statistical results when comparing the means are presented in Figure 3B. Both RNAi lines were confirmed to produce F1 progenies with reduced CG18549 expression, Figure 3A,B. However, for CG18549 RNAi line 1, CG18549 expression was reduced to 50\% compared with both controls, Figure 3A, while for the second CG18549 RNAi line (CG18549 RNAi line 2) CG18549 expression was measured to be reduced in both the RNAi control (90\% reduction) and the CG18549 knockdown (95\% reduction) flies, Figure 3A,B. Therefore, the second CG18549 RNAi line was not primarily used during the functional characterization.

\subsection{No Difference of F1 Progenies from RNAi Controls and CG18549 Knockdown Flies}

CG18549 is reported to be expressed during several embryo stages [36,37], but still its function remains unknown. Therefore, we hypothesized that it is a possibility that a knockdown of CG18549 could affect development and/or survival of the embryos. Hence, we wanted to study if there were any visible changes in appearance due to changes in developmental cues and/or alterations in the number of progenies that eclosed. The number of eclosed progenies were calculated for each experimental cross and the progenies were examined under a microscope. No differences were observed in appearance of the knockdown flies and the control flies (data not shown) and, in general, no significant differences were observed between the knockdowns and controls, or between the crosses, Figure 3C. However, the CG18549 RNAi line 1 was found to have a higher number of eclosed progenies compared with the Driver control and CG18549 knockdown flies, but the statistical power was not high enough, and the results could therefore not be statistically verified, but the trend was observed both in males and females. 

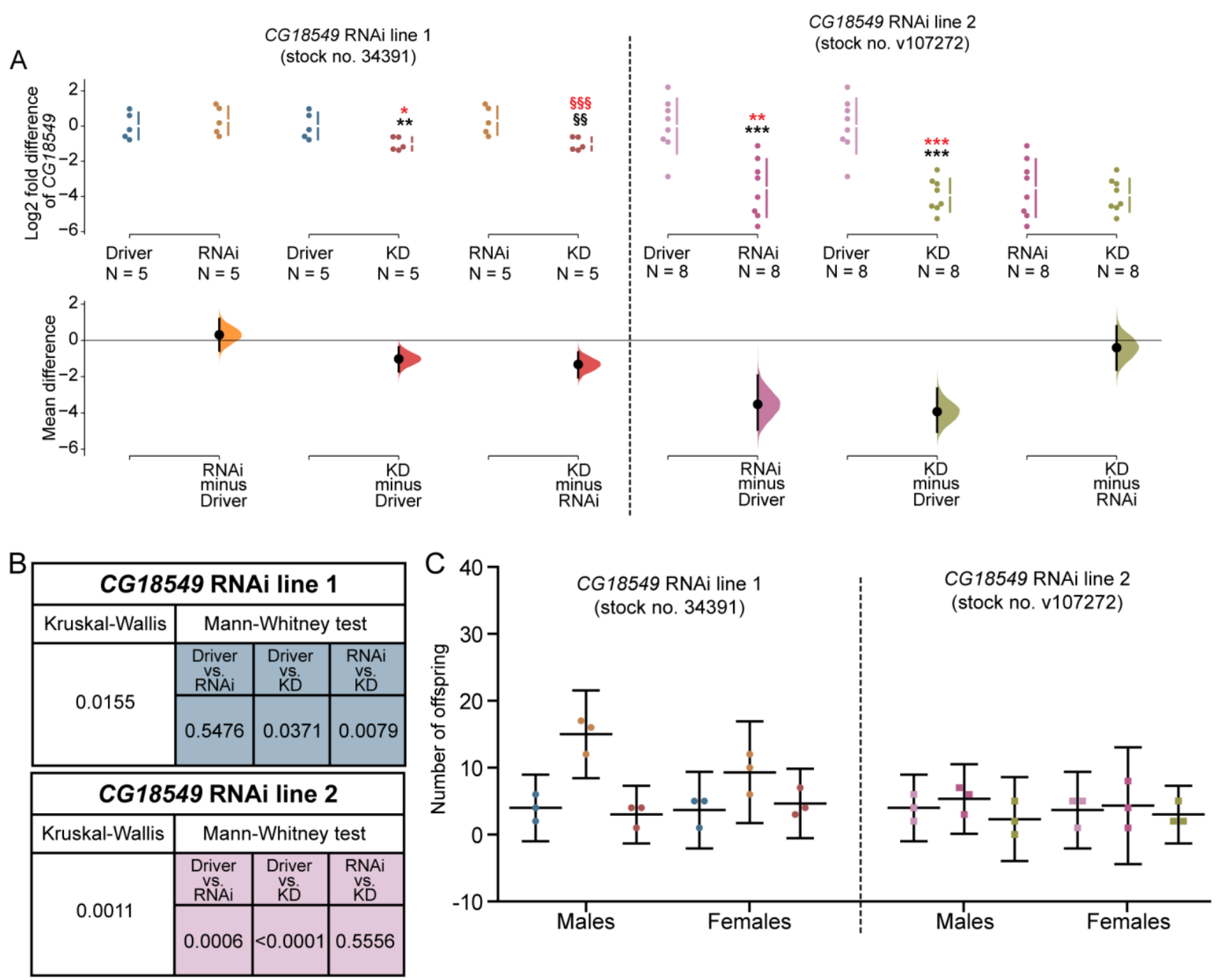

Figure 3. Validation of RNAi line functionality and counting of F1 progenies. CG18549 knockdown was verified for two CG18549 RNAi lines: $y[1]$ sc $\left[{ }^{*}\right] \mathrm{v}[1] ; \mathrm{P}\{\mathrm{y}[+\mathrm{t} 7.7] \mathrm{v}[+\mathrm{t} 1.8]$ = TRiP.HMS01385\}attP2 (CG18549 RNAi line 1, Stock no. 39341) and P\{KK102196\}VIE-260B (CG18549 RNAi line 2, Stock no. v107272) using the ubiquitous driver da-GAL4. Gene expression was measured with qPCR, $\log 2$ fold change was calculated according to the delta $\mathrm{Ct}$ method using three reference genes (Actin42a, Rpl11, Rp49), the Driver control was set as control. Kruskal-Wallis with Dunn's comparison against the control was used to initially analyze differences, exact p-values were calculated using multiple corrected Mann-Whitney as a post hoc test ${ }^{*}$ or $\S p<0.0491,{ }^{* *}$ or $\S \S p<0.0099,{ }^{* * *}$ or $\S \S \S p<0.0001{ }^{*}=$ against Driver control, $\S=$ against RNAi control). (A) The Log 2 fold mean differences for 6 comparisons are shown in the above Cumming estimation plot. The raw data is plotted on the upper axes; each mean difference is plotted on the lower axes as a bootstrap sampling distribution. Mean differences are depicted as dots; $95 \%$ confidence intervals are indicated by the ends of the vertical error bars. The effect sizes and CIs are reported above as: effect size [CI width lower bound; upper bound]. CG18549 RNAi line 1: Kruskal-Wallis $p=0.0155$, Mann-Whitney (MW) CG18549 knockdown * $p=0.0317$, $\S \S p=0.0079$, the unpaired mean difference between Driver ctrl and RNAi ctrl is 0.305 [95.0\% CI $-0.57,1.19], p$-value of the two-sided permutation $t$-test is 0.524; Driver ctrl and CG18549 knockdown is -1.02 [95.0\% CI $-1.71,-0.382]$, $p$-value of the two-sided permutation $t$-test is 0.021 ; RNAi ctrl and CG18549 knockdown is -1.33 [95.0\% CI -2.05, -0.659], $p$-value of the two-sided permutation $t$-test is 0.0004 . CG18549 RNAi line 2: Kruskal-Wallis < 0.0001, MW CG18549 knockdown $p \leq 0.0001$, RNAi ctrl $p=0.0006$, the unpaired mean difference between Driver ctrl and RNAi ctrl is -3.53 [95.0\% CI $-4.93,-1.94]$, $p$-value of the two-sided permutation $t$-test is 0.0012; Driver ctrl and CG18549 knockdown is -3.93 [95.0\% CI $-5.05,-2.65], p$-value of the two-sided permutation $t$-test is 0.0006; RNAi ctrl and CG18549 knockdown is -0.404 [95.0\% CI -1.62, 0.789], $p$-value of the two-sided permutation $t$-test is 0.556. (in red effect size differences ${ }^{*}=$ significant difference against the Driver control, $\S=$ significant difference against the RNAi control). (B) Summary of $p$-values in the statistical analysis. (C) F1 progenies were counted from both RNAi lines and controls, the males and females of F1 progenies are shown in the above scatter plot. 


\subsection{CG18549 Expression Is Affected by the Concentration of Sugar and Protein in the Food}

Several of the MFSD proteins are known to be involved in the transport of important metabolites such as glucose, amino acids, and fats $[12,16]$. Furthermore, earlier studies on CG18549 [20], as well as the mouse orthologue Mfsd11 [17], reported that the gene expression is affected by nutrient availability. So far, the findings in D. melanogaster were observed on samples obtained from the whole-body of the fly, while the data from mice were measured in brain sections. Here, we wanted to focus on the expression in the heads from flies to be able to compare the results from the brains in mice. We hypothesized that we would observe gene alterations as a response to different diets similar to those reported earlier. To test this, the relative mRNA expression of CG18549 was measured in five- to seven-days-old wildtype (CSORC) flies that were fed diets with different ratios of sugar (S) and protein (Y), Figure 4. CG18549 was indeed found to be altered in the head tissue. The expression was increased in flies fed a 40:40 g sugar:yeast (S:Y) diet and a 10:40 g S:Y diet, which is similar to what have been reported in mice fed a high-fat diet [17]; while the gene expression was reduced in flies fed 2.5:40 g S:Y and 40:10 g S:Y, Figure 4A. The reduction in flies fed 40:10 g S:Y corresponds to what has been previously reported in flies [20]. However, no difference was measured in flies fed 2.5:2.5 g S:Y, which do not correspond to the reported difference in CG18549 expression observed in whole-body tissues of flies [20]. Together these results strongly suggest that nutrient concentrations such as sugar (sucrose) and protein (yeast) have a biological effect on the expression of CG18549.

Earlier findings in body focused on the sugar content in food; hence, some information regarding gene expression alterations as a response to fluctuating sugar and protein ratios is missing. The changes observed for both tissues vary, most likely due to the difference in how the data is presented. Therefore, we wanted to convert the data collected from the whole-body of flies [20] to $\log 2$ fold to be able to make a better comparison between the fly tissues from the body and head, Figure 4B. No difference between the control diet, that is the 10:10 g S:Y, was found, but interestingly CG18549 was highly altered with a large difference of the means of the samples in the whole-body tissue of flies fed 2.5:2.5 g S:Y diets and no differences were observed in the head tissue sample of flies fed 2.5:2.5 g S:Y. Furthermore, a large difference between the CG18549 mRNA expression means of the whole-body tissue samples and the head tissue samples obtained from flies fed the 40:10 g S:Y diet was detected, but still these samples were regulated in similar patterns compared with the controls for each tissue, Figure 4B. These results conclude that there are similarities in how the CG18549 expression is altered in whole-body tissue and head tissue in flies subjected to a high sugar diet and normal diet. However, the results also strongly suggest that the body CG18549 is more affected after a calorie deficient diet compared with the heads where the CG18549 expression instead is highly affected by a sugar rich diet, which could be due to the difference in nutrient need and storage within the body compared to the head (brain).

\subsection{Starvation Does Not Affect the CG18549 in the Heads of Wildtype Flies}

To further elaborate on the involvement of CG18549 in metabolic pathways and cues, alterations in gene expression in flies subjected to complete starvation was investigated. Previous findings have shown that CG18549 is affected by starvation and that the expression normalized upon refeeding [20]. However, once again the gene expression was measured in whole-body tissue samples, and since a difference in gene alterations was observed between whole-body tissue and head tissue, we wanted to investigate if it was also true in flies subjected to starvation. To do so, the gene expression of CG18549 was measured in five- to seven-days-old flies subjected to complete starvation. No differences were observed between the timepoints $(0,3,6,9,12$ and $24 \mathrm{~h}$ of starvation), Figure $4 \mathrm{C}$.

In the body, upregulation of CG18549 has been reported after $3 \mathrm{~h}$ of starvation [20]. In the heads, one can observe a trend towards upregulation, but no statistical significance was reported. Once again, the data from the whole-body tissue were not presented in log2 fold change and therefore the data from these samples were collected and recalculated to 
be able to perform a better comparison to the head tissue samples. After the conversion to $\log 2$ fold change, the differences were still present in the samples obtained from the whole-body. Interestingly, the $\log 2$ fold expression in whole-body tissue and head tissue were found to follow a similar pattern and the means of the control and the $3 \mathrm{~h}$ starved flies were similar, Figure 4D. Taken together, these results suggest that CG18549 is not as affected by starvation in the head of adult flies, but since similar means were observed between the tissues, it is most likely that the difference in outcome was due to variations in the number of samples (body $0 \mathrm{~h} n=6$ and $3 \mathrm{~h} \mathrm{n}=8$; head $0 \mathrm{~h} \mathrm{n}=5$ and $3 \mathrm{~h} \mathrm{n}=5$ ).

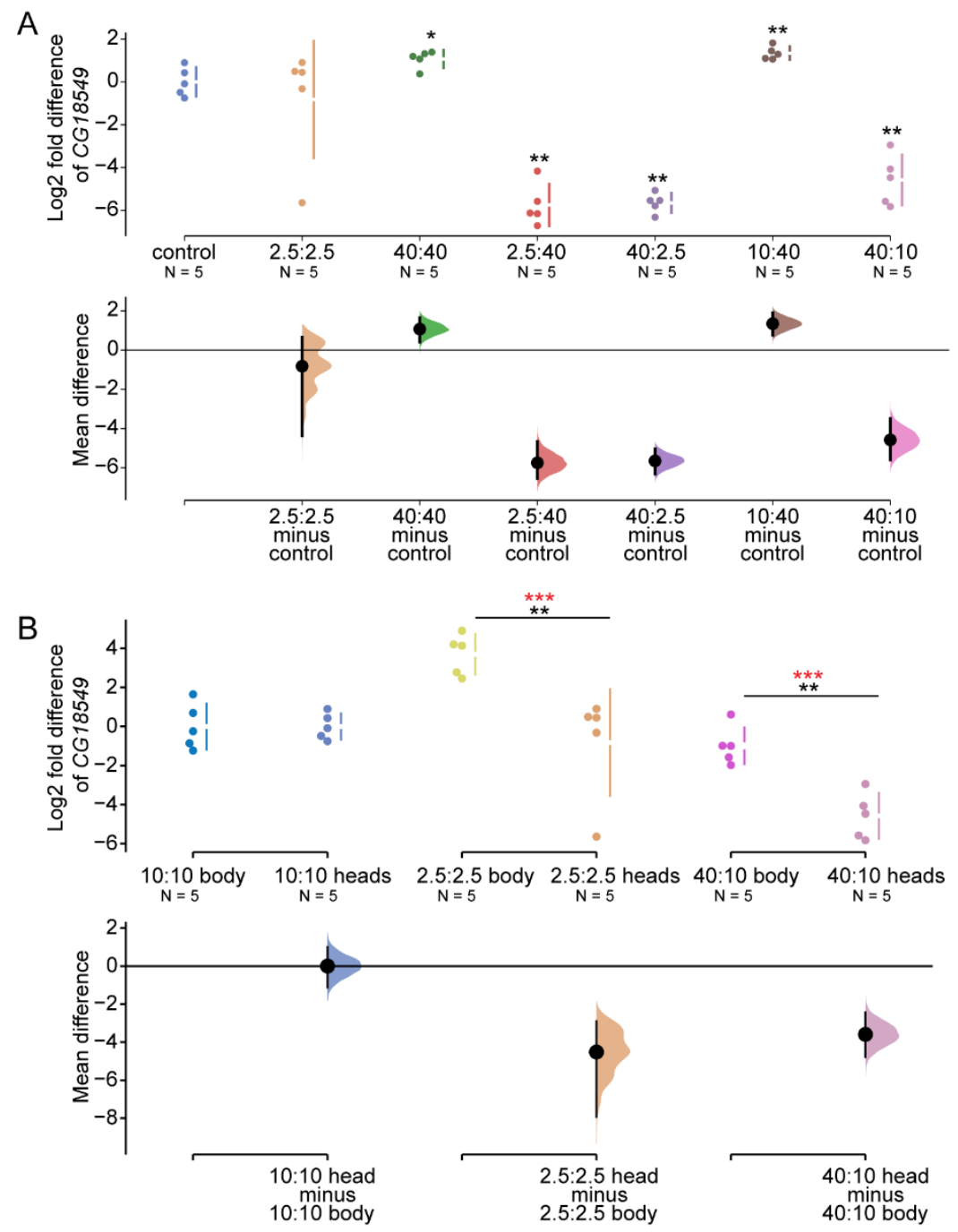

C
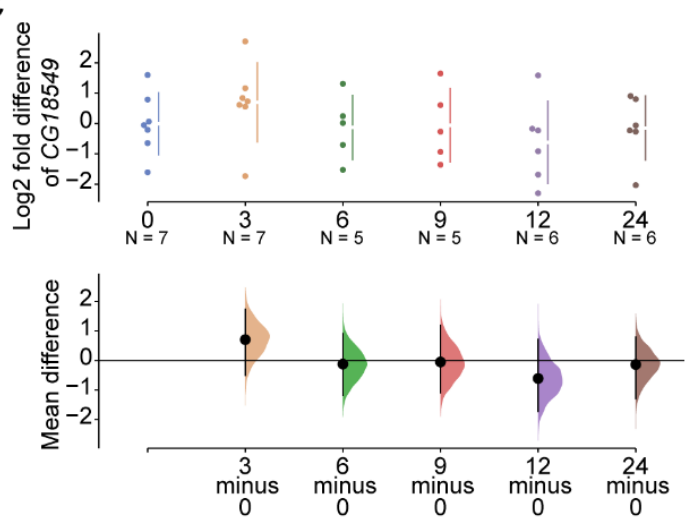

Figure 4. Cont. 


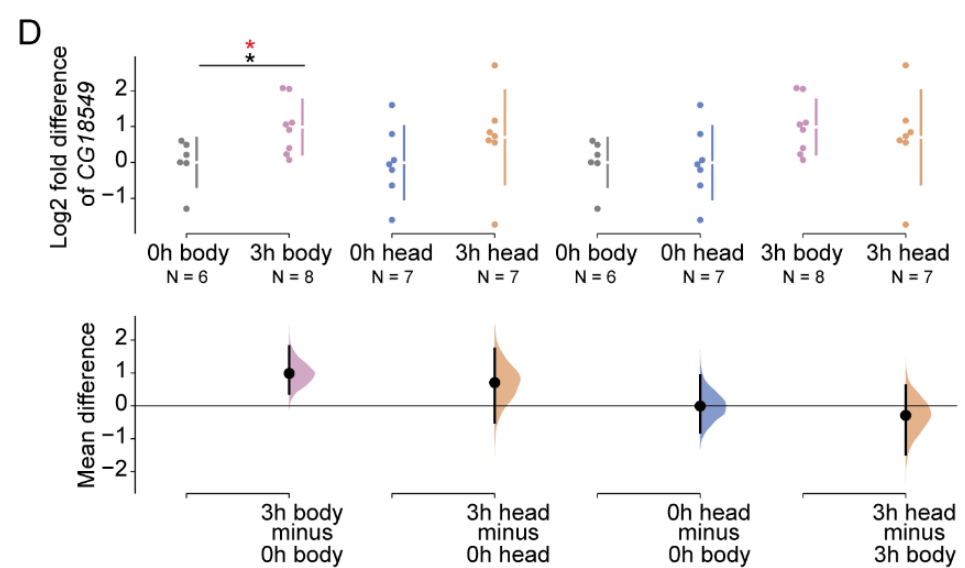

Figure 4. CG18549 expression alterations as a response to sugar and protein imbalanced diets, and starvation. qPCR was used to measure the gene expression of CG18549 in heads from five- to sevendays-old CSORC flies $(\mathrm{n}=5)$ fed diets with different sugar and yeast ratio: 10:10 g/dL (control), 2.5:2.5 g/dL, 40:40 g/dL, 2.5:40 g/dL, 10:40 g/dL, 40:2.5 g/dL and 40:10 g/dL diets or subjected to 0 $(n=7), 3(n=7), 6(n=5), 9(n=5), 12(n=6)$ and $24(n=6)$ hours of starvation. The log 2 fold change was calculated with the delta Ct method against three reference genes (Actin42a, Rpl11, Rp49), the Driver control was set as control. Kruskal-Wallis with Dunn's comparison against the control was used to initially analyze differences, exact $p$-values were calculated using multiple corrected MannWhitney as a post hoc test ${ }^{*} p<0.0489,{ }^{* *} p<0.0099,{ }^{* * *} p<0.0001$. (A) Log2 fold mean differences of CG18549 for 6 comparisons against the shared control 10:10 g/dL sugar:yeast are shown in the a Cumming estimation plot. The raw data is plotted on the upper axes. On the lower axes, mean differences are plotted as bootstrap sampling distributions. Each mean difference is depicted as a dot. Each $95 \%$ confidence interval is indicated by the ends of the vertical error bars. The effect sizes and CIs are reported above as: effect size [CI width lower bound; upper bound]. Kruskal-Wallis $p=0.0001$, the unpaired mean difference between control and 2.5:2.5 g/dL is Mann-Whitney (MW) $p=0.6905$, effect size -0.822 [95.0\% CI $-4.38,0.664], p$-value of the two-sided permutation t-test is 0.822; control and 40:40 g/dL is MW $p=0.0317$, effect size 1.07 [95.0\% CI 0.408, 1.65], $p$-value of the two-sided permutation $t$-test is 0.019 ; control and 2.5:40 g/dL is MW $p=0.0079$, effect size -5.75 [95.0\% CI $-6.55,-4.66], p$-value of the two-sided permutation $t$-test is 0.0 ; control and $40: 2.5 \mathrm{~g} / \mathrm{dL}$ is MW $p=0.0079$, effect size -5.65 [95.0\% CI $-6.32,-5.04], p$-value of the two-sided permutation $t$-test is 0.003 ; control and 10:40 g/ dL is MW $p=0.0079$, effect size 1.35 [95.0\% CI 0.758, 1.91], $p$-value of the two-sided permutation $t$-test is 0.0022 ; control and 40:10 g/ $\mathrm{dL}$ is MW $p=0.0079$, effect size -4.58 [95.0\% CI $-5.59,-3.49], p$-value of the two-sided permutation $t$-test is 0.0018 . Previous published data [20] was, with permission of all authors, reanalyzed and plotted against the results obtained from head tissue. (B) CG18549 expression in body and head: 10:10 g/dL, 2.5:2.5 g/dL and 40:10 $\mathrm{g} / \mathrm{dL}$. The mean differences for 3 comparisons are shown in the above Cumming estimation plot with same settings as in (B). Kruskal-Wallis $p=0.0004$, the unpaired mean difference between 10:10 $\mathrm{g} / \mathrm{dL}$ in body and 10:10 $\mathrm{g} / \mathrm{dL}$ in head is MW $p=0.8413$, effect size $1.4 \times 10^{-6}[95.0 \% \mathrm{CI}-1.13,1.0]$, $p$-value of the two-sided permutation $t$-test is $0.997 ; 2.5: 2.5 \mathrm{~g} / \mathrm{dL}$ in body and $2.5: 2.5 \mathrm{~g} / \mathrm{dL}$ in head is MW $p=0.0079$, effect size -4.52 [95.0\% CI $-7.92,-2.9], p$-value of the two-sided permutation $t$-test is $0.0 ; 40: 10 \mathrm{~g} / \mathrm{dL}$ in body and $40: 10 \mathrm{~g} / \mathrm{dL}$ in head is MW $p=0.0079$, effect size -3.59 [95.0\% CI -4.78 , -2.44], $p$-value of the two-sided permutation $t$-test is 0.0 . (C) The mean Log2 fold differences of CG18549 for 5 comparisons against the shared control 0 are shown in the above Cumming estimation plot. The raw data is plotted on the upper axes. On the lower axes, mean differences are plotted as bootstrap sampling distributions. Each mean difference is depicted as a dot. Each $95 \%$ confidence interval is indicated by the ends of the vertical error bars. The effect sizes and CIs are reported above as: effect size [CI width lower bound; upper bound]. Kruskal Wallis $p=0.5194$, the unpaired mean difference between 0 and 3 is 0.705 [95.0\% CI $-0.505,1.74], p$-value of the two-sided permutation t-test is $0.269 ; 0$ and 6 is -0.124 [95.0\% CI $-1.19,0.912], p$-value of the two-sided permutation $t$-test is $0.844 ; 0$ and 9 is -0.0533 [ $95.0 \% \mathrm{CI}-1.1,1.19]$, $p$-value of the two-sided permutation $t$-test is 0.928 ; 
0 and 12 is -0.611 [95.0\% CI $-1.73,0.716], p$-value of the two-sided permutation $t$-test is $0.366 ; 0$ and 24 is -0.139 [95.0\% CI $-1.29,0.793], p$-value of the two-sided permutation $t$-test is 0.804 . Previous published data [20] was, with permission of all authors, reanalyzed and plotted against the results obtained from head tissue. (D) CG18549 expression in body and head: $0 \mathrm{~h}$ and $3 \mathrm{~h}$ of starvation, the mean difference for 4 comparisons of CG18549 expression are shown in the above Cumming estimation plot. The effect sizes and CIs are reported above as: effect size [CI width lower bound; upper bound]. Kruskal-Wallis $p=0.0325$, the unpaired mean difference between $0 \mathrm{~h}$ body and $3 \mathrm{~h}$ body is MW $p=0.0293$, effect size 0.987 [95.0\% CI 0.371, 1.81], $p$-value of the two-sided permutation $t$-test is $0.0248 ; 0 \mathrm{~h}$ head and $3 \mathrm{~h}$ head is MW $p=0.2593$, effect size 0.705 [95.0\% CI $-0.505,1.74]$, $p$-value of the two-sided permutation $t$-test is $0.268 ; 0 \mathrm{~h}$ body and $0 \mathrm{~h}$ head is MW $p=0.7308$, effect size -0.0074 [95.0\% CI $-0.806,0.931], p$-value of the two-sided permutation $t$-test is $0.988 ; 3 \mathrm{~h}$ body and $3 \mathrm{~h}$ head is MW $p=0.8665$, effect size -0.29 [95.0\% CI $-1.48,0.622]$, $p$-value of the two-sided permutation $t$-test is 0.622 .

\subsection{CG18549 Knockdown Affects the Expression of Glucagon-like Genes}

CG18549 has been shown to be expressed in metabolic active tissues such as the fat bodies and salivary glands [35]. Together with the earlier findings about transcription binding sites linked to metabolism in the promoter region of mouse $M f_{s} d 11$ [20] as well as the altered gene expression as a response to nutrient levels, led us to the theory that CG18549 knockdown possibly affects metabolic cues. To study this, we investigated alterations in gene expression of glucagon and insulin-like peptide and their respective receptor. The expression of $A k h, A k h R, I l p 2$, Ilp3, Ilp5, Ilp6 and InR were measured in wholebody tissue samples of five- to seven-days-old Driver control (da-GAL4), RNAi control and CG18549 knockdown flies (CG18549 RNAi line 1), Figure 5A,B. Differences in expression were observed between the CG18549 knockdown flies and both Driver and RNAi control flies for $A k h$ (upregulated) and $A k h R$ (downregulated). Expression differences against the Driver control were also measured for Ilp5, Ilp6 and InR, Figure 5A,B. No differences were observed for Ilp2 and Ilp3, Figure 5A. Interestingly, the results of the RNA sequencing, Table 2 and Supplementary Data sheet 1, on the siblings from the same three crosses did only display similar alterations in gene expression of $A k h$ and $A k h R$, suggesting that the changes in the insulin-like peptides and the insulin receptor observed from the quantitative PCR are secondary effects depending on other factors than the knockdown.

The largest effects were measured for $A k h$ and $A k h R$; hence, these two genes were also run on the second CG18549 RNAi line (CG18549 RNAi line 2), Figure 5C, to study if the alterations were also present; hence, this strengthens the conclusion that CG18549 affects this hormone and its receptor. Gene expression of CG18549 in adult male flies were measured and an alteration of $A k h$ were found between the RNAi control and the Driver as well as between the RNAi control and CG18549 knockdown, while no differences were found for $A k h R$, Figure $5 C$. This suggests that either there are line specific effects observed or that the effects on glucagon and insulin-like linked genes are secondary and not a result of the CG18549 knockdown. The mRNA levels are constantly fluctuating, which means that the alterations of the glucagon genes could rather be due to the time point of food intake of the flies.

\subsection{CG18549 Knockdown Flies Are Equally Resistant to Starvation as the Controls}

The gene expression of CG18549 has been shown to be affected by starvation ([20], while we observe no change in gene expression when limiting the study to the head. Therefore, we wanted to investigate starvation further, to study if the starvation resistance of the CG18549 knockdown flies was affected, Figure 5 and Table 3. The driver line used for the starvation resistance assay was the da-GAL4 line. Five- to seven-days-old male flies were subjected to starvation in drosophila activity monitor systems (DAMS) where activity (beam breaks) was monitored and the last beam break of each fly was determined as the time point of death. The average last beam break of CG18549 knockdown flies was 
similar to the average last beam break of the Driver control flies, but a little higher than the last beam break of the RNAi control flies. A difference between the two controls were also monitored, Figure 5D. In addition, the survival proportions were plotted to be able to study the death curves more in detail and for instance see if they are similar or for example biphasic. When comparing the survival proportions over time the RNAi control flies were observed to be more sensitive to starvation, but also the CG18549 knockdown flies deceased faster compared with Driver control flies, Figure 5E and Table 3. In addition, the activity during starvation was plotted to study if the difference in starvation resistance could be due to differences in activity, which could indicate if the difference in starvation resistance is due to burning their nutrient storages at different paces, Figure 5F. The locomotion plot revealed that the RNAi control flies move more compared with both Driver control and CG18549 knockdown flies, suggesting that the sensitivity to starvation could be that they use their nutrient storage faster and hence decease faster. However, in future experiments, measurements of nutrient storage and depletion are needed to validate this finding.
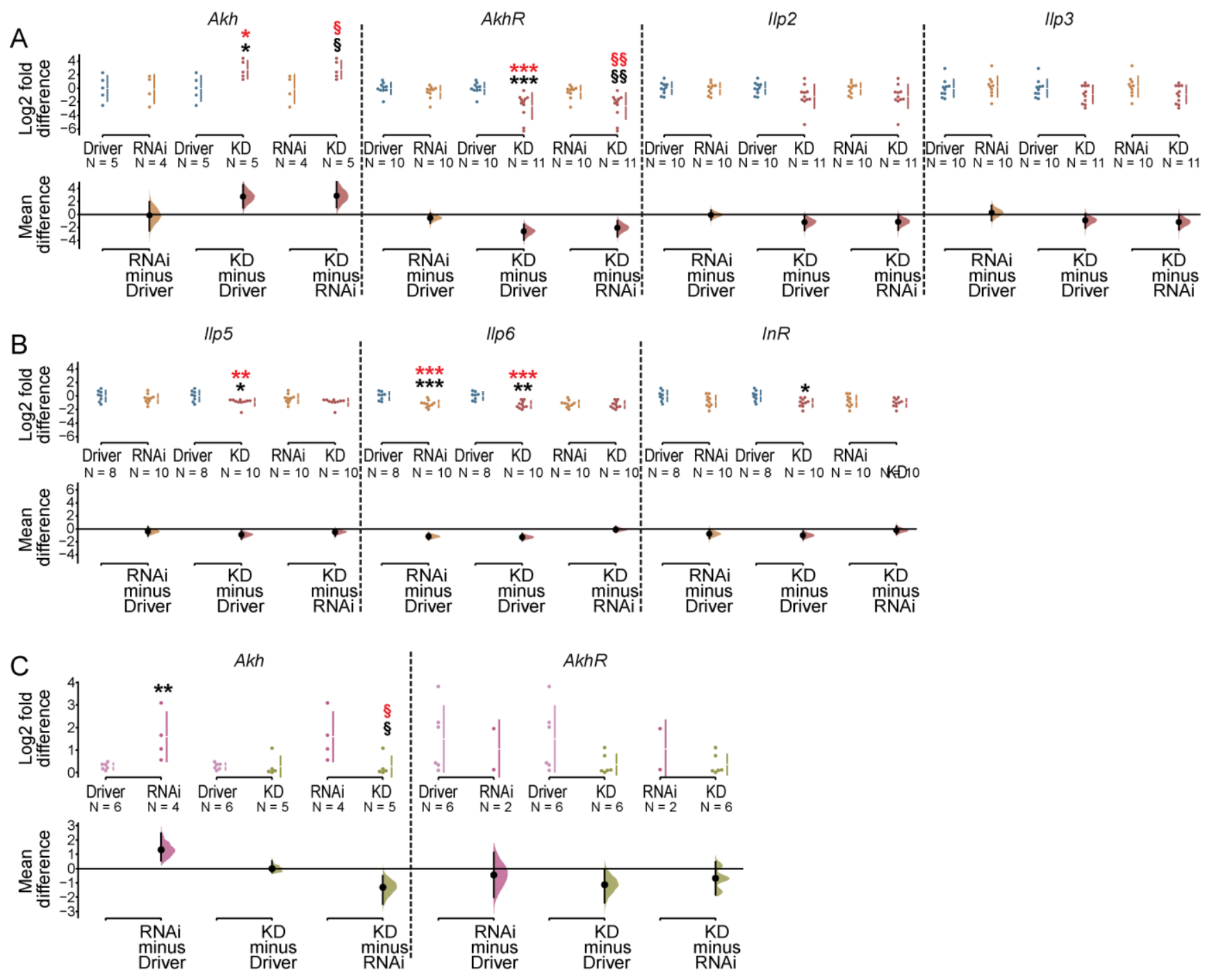

Figure 5. Cont. 


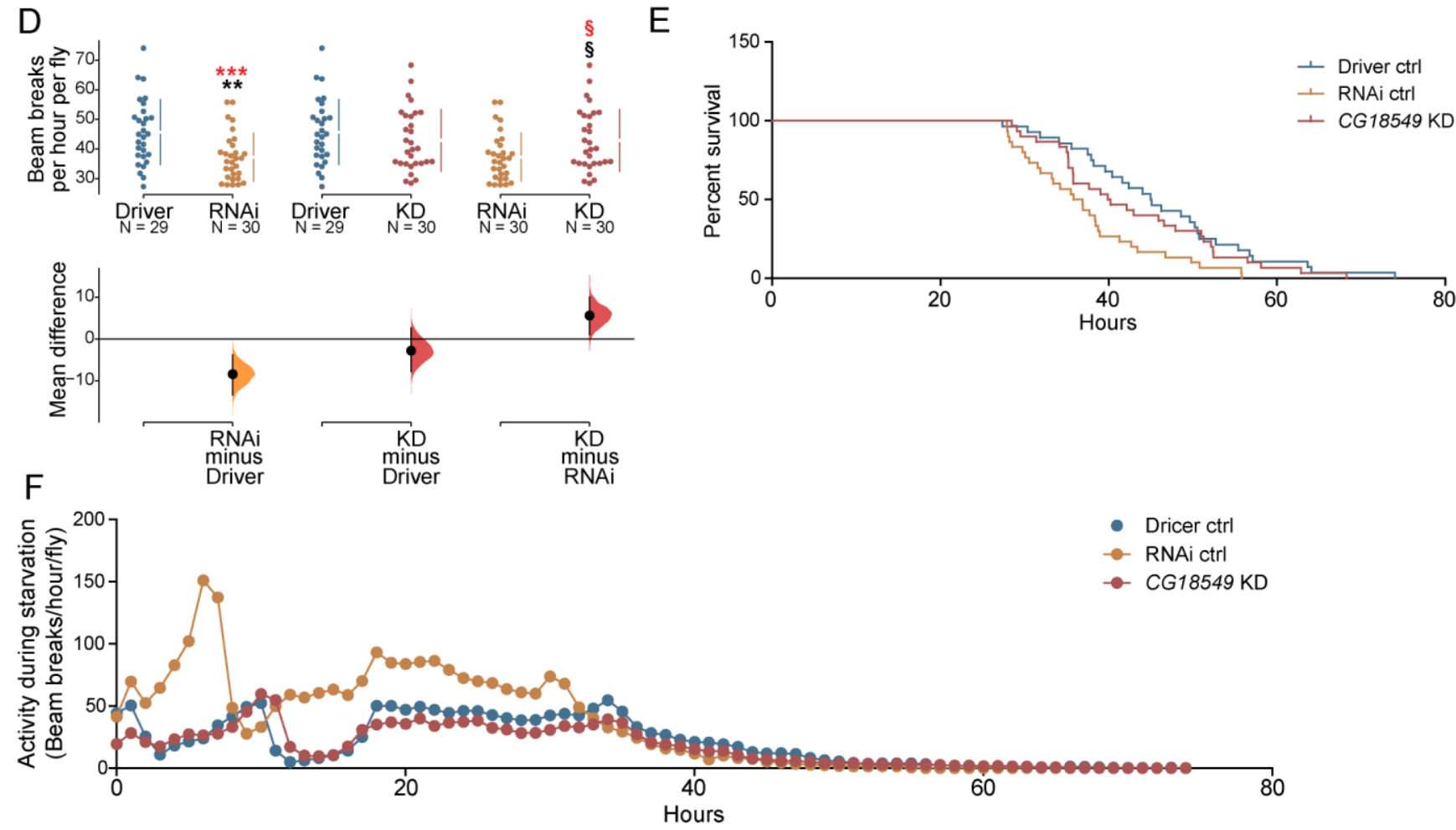

Figure 5. mRNA expression of glucagon and insulin like peptides and receptors, and starvation resistance in CG18549 knockdown flies. The ubiquitous da-GAL4 driver, $\mathrm{w}^{1118}$ and CG18549 RNAi line 1 and 2 were used. Gene expression of Akh, AkhR, Ilp2, Ilp3, Ilp5, Ilp6 and InR were measured with qPCR, $\log 2$ fold changes were calculated according to the delta Ct method using three reference genes (Actin42a, Rpl11, Rp49) for CG18549 RNAi line 1 and controls, while one reference gene (Actin42a) was used for CG18549 RNAi line 2 and controls. The Driver control was set as control. Kruskal-Wallis with Dunn's comparison against the control was used to initially analyze differences, exact $p$-values were calculated using multiple corrected Mann-Whitney as a post hoc test * or $\S p<0.0491$, ${ }^{* *}$ or $\S \S p<0.0099$, ${ }^{* * *}$ or $\S \S \S p<0.0001$ (in red effect size differences $*$ = significant difference against the Driver control, $\S=$ significant difference against the RNAi control). (A,B) Data of CG18549 RNAi line 1 (* = significant difference against the Driver control, $\S$ = significant difference against the RNAi control. The mean differences for 21 comparisons are shown in the above Cumming estimation plot. The raw data is plotted on the upper axes; each mean difference is plotted on the lower axes as a bootstrap sampling distribution. Mean differences are depicted as dots; $95 \%$ confidence intervals are indicated by the ends of the vertical error bars: $A k h$ (RNAi ctrl minus Driver ctrl -0.127 [95.0\% CI -2.48, 1.95] $p=0.886$; CG18549 knockdown minus Driver ctrl 2.75 [95.0\% CI $1.03,4.6] p=0.0254 ;$ CG18549 knockdown minus RNAi ctrl $2.88[95.0 \%$ CI 1.07, 5.16] $p=0.0268$ ); AkhR (RNAi ctrl minus Driver ctrl -0.55 [95.0\% CI -1.34 p=0.197; CG18549 knockdown minus Driver ctrl -2.6 [95.0\% CI -3.96, - 1.56] $p=0.0006$; CG18549 knockdown minus RNAi ctrl is -2.05 [95.0\% CI -3.42, -0.971] $p=0.0036$ ); Ilp2 (RNAi ctrl minus Driver ctrl $-0.058[95.0 \%$ CI $-0.799,0.665] p=0.888$; CG18549 knockdown minus Driver ctrl -1.19 [95.0\% CI $-2.46,-0.223] p=0.0542$; CG18549 knockdown minus RNAi ctrl -1.13 [95.0\% CI -2.4, -0.166] $p=0.0656$ ); Ilp3 (RNAi ctrl minus Driver ctrl 0.263 $[95.0 \%$ CI $-0.946,1.41] p=0.675 ;$ CG18549 knockdown minus Driver ctrl $-0.904[95.0 \%$ CI $-2.05,0.0776] p=0.126 ;$ CG18549 knockdown minus RNAi ctrl -1.17 [95.0\% CI - 2.33, -0.051]. $p=0.0712$ ); Ilp5 (RNAi ctrl minus Driver ctrl -0.406 [95.0\% CI -1.05, 0.281] $p=0.266$; CG18549 knockdown minus Driver ctrl -0.907 [95.0\% CI $-1.55,-0.339] p=0.0098 ;$ CG18549 knockdown minus RNAi ctrl -0.501 [95.0\% CI -1.12, -0.068] $p=0.0946$ ); Ilp6 (RNAi ctrl minus Driver ctrl is -1.16 [95.0\% CI -1.64, -0.667] $p=0.001 ; C G 18549$ knockdown minus Driver ctrl -1.28 [95.0\% CI -1.75, -0.749] $p=0.0004 ;$ CG18549 knockdown minus RNAi ctrl -0.117 [95.0\% CI -0.519, 0.318] $p=0.615$ ); InR (RNAi ctrl minus Driver ctrl 0.777 [95.0\% CI $-1.47,-0.0428] p=0.0628 ;$ CG18549 knockdown minus Driver ctrl -1.0 [95.0\% CI -1.61, -0.375] $p=0.0084 ;$ CG18549 knockdown minus RNAi ctrl -0.223 [95.0\% CI -0.8, 0.415] $p=0.503$ ); Akh (up, Kruskal-Wallis $p=0.0340$, Mann-Whitney $(\mathrm{MW}) * p=0.0317, \S p=0.0317)$, AkhR (down, Kruskal-Wallis $p=0.0005, \mathrm{MW} * p=0.0006, \S p=0.0035)$, Ilp2 (Kruskal-Wallis $p=0.1398$ ), Ilp3 (Kruskal-Wallis $p=0.2033)$, Ilp5 (CG18549 knockdown, down, Kruskal-Wallis $\left.p=0.0566, \mathrm{MW}^{*} p=0.0367\right)$, Ilp6 (CG18549 knockdown, down, Kruskal-Wallis $p=0.0015, \mathrm{MW}^{*} p=0.0019$; RNAi control, down * $\left.p=0.0009\right)$ and InR 
(CG18549 knockdown, down, Kruskal-Wallis $p=0.0395, \mathrm{MW} * p=0.0117$ ). (C) Data of CG18549 RNAi line 2; Log2 fold change of CG18549 in Driver control, RNAi control and CG18549 knockdown flies ${ }^{*}=$ significant difference against the Driver control, $\S=$ significant difference against the RNAi control, in red effect size differences * ${ }^{*}$ significant difference against the Driver control, $\S=$ significant difference against the RNAi control), Cumming estimation plot illustrating the effects size with the same setup as in A and B. Akh: Kruskal-Wallis $p=0.0064$, effect size RNAi ctrl minus Driver ctrl 1.31 [95.0\% CI 0.552, 2.47] $p=0.0914$; CG18549 knockdown minus Driver ctrl -0.00567 [95.0\% CI $-0.264,0.535] p=0.984$; CG18549 knockdown minus RNAi ctrl -1.31 [95.0\% CI -2.49, -0.497] $p=0.0264 ;$ AkhR: Kruskal-Wallis $p=0.1819$, effect size RNAi ctrl minus Driver ctrl -0.45 [95.0\% CI -2.02, 1.13] $p=0.6$, CG18549 knockdown minus Driver ctrl -1.13 [95.0\% CI $-2.4,-0.118] p=0.103$, CG18549 knockdown minus RNAi ctrl -0.678 [95.0\% CI -1.84, 0.47], $p=0.0734): A k h\left({ }^{*} p=0.0095\right.$, $\S p=0.05)$, AhkR (no change). (D-F) Five- to seven-days-old male flies from Driver control, RNAi control and CG18549 knockdown (da-GAL4 driver, $\mathrm{n}=30$ ) were transferred to glass tubes, prepared with $1 \%$ agarose, and contained in the DAMS until the last beam break. Last beam crossing per fly was defined as the timepoint of death. Differences were calculated using Kruskal-Wallis with Dunn's comparison and/or Mann-Whitney as a post hoc test to calculate exact $p$-values ${ }^{*} p<0.0492$, ${ }^{* *} p<0.0099,{ }^{* * *} p<0.0001{ }^{*}=$ significant difference against the Driver control, $\S=$ significant difference against the RNAi control, in red effect size differences * = significant difference against the Driver control, $\S=$ significant difference against the RNAi control). (D) The mean difference of starvation resistance is presented in the above Cumming estimation plot. The raw data is plotted on the upper axes; each mean difference is plotted on the lower axes as a bootstrap sampling distribution. Mean differences are depicted as dots; $95 \%$ confidence intervals are indicated by the ends of the vertical error bars. The unpaired mean difference between Driver ctrl and RNAi ctrl is -8.41 [95.0\% CI $-13.4,-3.82], p$-value of the two-sided permutation $t$-test is 0.0006; Driver ctrl and CG18549 knockdown is -2.78 [95.0\% CI -7.8, 2.64], $p$-value of the two-sided permutation $t$-test is 0.321; RNAi ctrl and CG18549 knockdown is 5.63 [95.0\% CI 1.01, 10.1], $p$-value of the two-sided permutation t-test is 0.0206. (E) A survival proportion plot, Log-rank (Mantel-Cox) test (conservative) $p=0.0040$, and $(\mathbf{F})$ ) a point-connection line graph to illustrate the survival over time and locomotion.

Table 3. Survival proportions of the Driver control (blue column, $d a-G A L 4>w 1118$ ), the RNAi control (yellow column, w1118 > CG18549 RNAi line 1) and the CG18549 knockdown (red column, da-GAL4 > CG18549 RNAi line 1) flies that were used in the starvation resistance assay. The percentage together with the upper and lower $95 \% \mathrm{CI}$ for each line at specific hours are presented. A grey row indicates the hours that a line deceased of starvation.

\begin{tabular}{|c|c|c|c|c|c|c|c|c|c|}
\hline \multicolumn{10}{|c|}{ Survival Proportions } \\
\hline \multirow{2}{*}{ Hours } & \multicolumn{3}{|c|}{ Driver Control } & \multicolumn{3}{|c|}{ RNAi Control } & \multicolumn{3}{|c|}{ CG18549 Knockdown } \\
\hline & Percentage & +Error & -Error & Percentage & +Error & -Error & Percentage & +Error & -Error \\
\hline 0.00 & 100 & & & 100 & & & 100 & & \\
\hline 27.36 & 96.43 & 3.06 & 19.18 & & & & & & \\
\hline 27.91 & & & & 96.67 & 2.86 & 18.06 & & & \\
\hline 27.95 & & & & 93.33 & 4.96 & 17.45 & & & \\
\hline 28.00 & & & & 90.00 & 6.66 & 17.88 & & & \\
\hline 28.20 & & & & 86.67 & 8.11 & 18.39 & & & \\
\hline 28.48 & & & & & & & 96.67 & 2.86 & 18.06 \\
\hline 28.53 & & & & 83.33 & 9.37 & 18.84 & & & \\
\hline 29.11 & & & & & & & 93.33 & 4.96 & 17.45 \\
\hline 29.50 & & & & & & & 90.00 & 6.66 & 17.88 \\
\hline 29.75 & & & & 80.00 & 10.48 & 19.20 & & & \\
\hline 30.07 & & & & 76.67 & 11.46 & 19.46 & & & \\
\hline 30.37 & 92.86 & 5.31 & 18.51 & & & & & & \\
\hline 30.53 & & & & 73.33 & 12.34 & 19.64 & & & \\
\hline 31.38 & & & & & & & 86.67 & 8.12 & 18.39 \\
\hline 31.53 & & & & 70.00 & 13.12 & 19.74 & & & \\
\hline 31.82 & 89.29 & 7.13 & 18.93 & & & & & & \\
\hline 31.87 & & & & 66.67 & 13.81 & 19.75 & & & \\
\hline 33.23 & & & & 63.33 & 14.42 & 19.69 & & & \\
\hline 33.4 & & & & 60.00 & 14.95 & 19.55 & & & \\
\hline 34.1 & 85.71 & 8.67 & 19.42 & & & & & & \\
\hline 34.13 & & & & & & & 83.33 & 9.37 & 18.84 \\
\hline 34.23 & & & & 56.67 & 15.41 & 19.34 & & & \\
\hline 34.98 & & & & & & & 80 & 10.48 & 19.20 \\
\hline
\end{tabular}


Table 3. Cont.

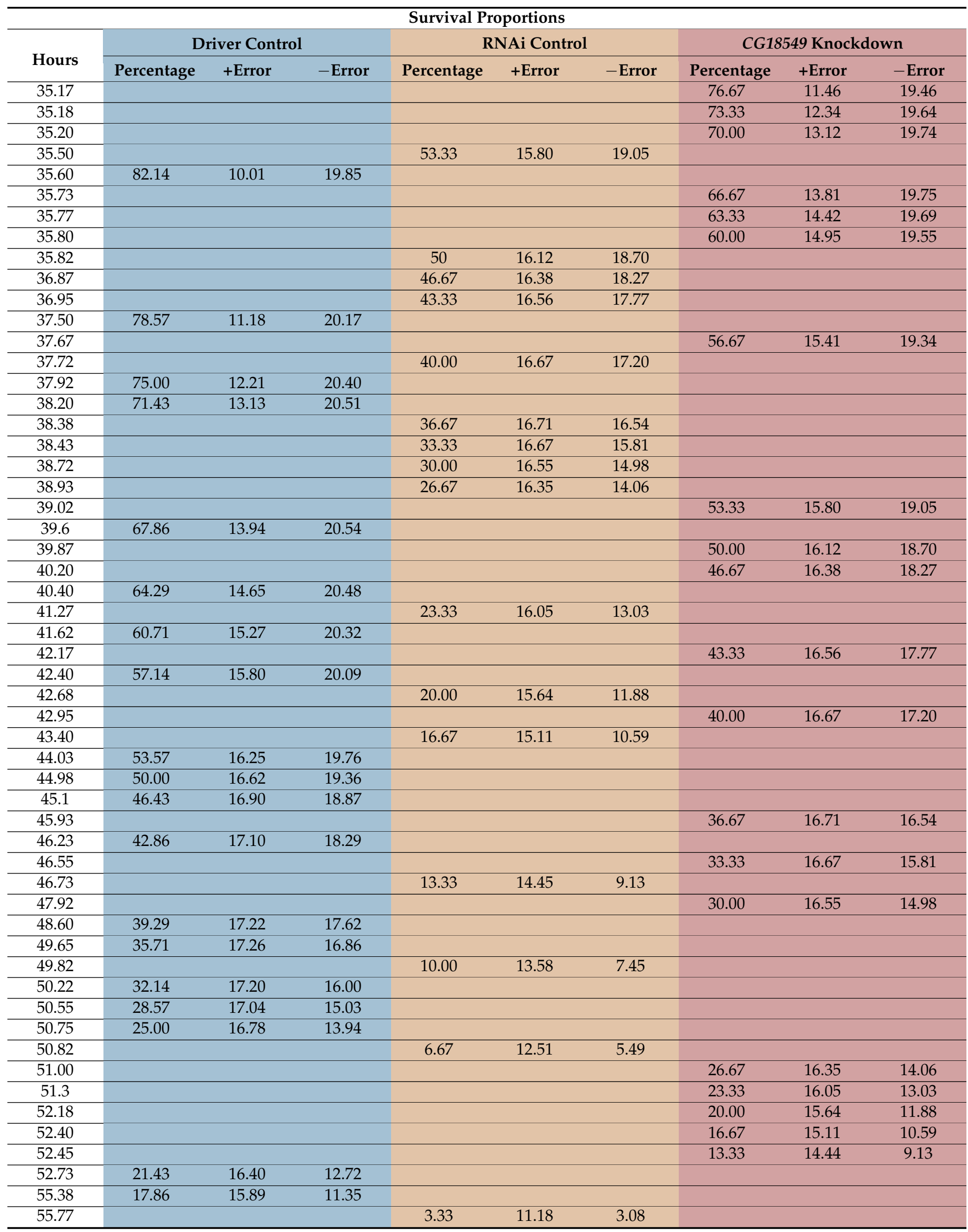


Table 3. Cont

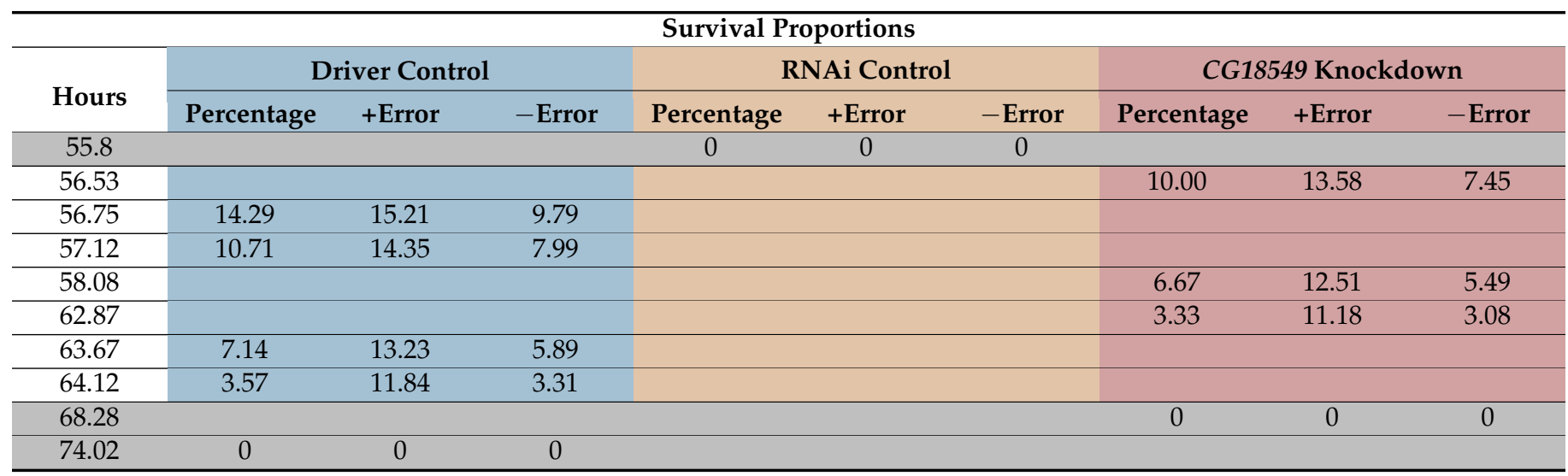

\subsection{Ubiquitous Knockdown of CG18549 Has a Small Effect on Locomotion}

Since CG18549 expression is affected by nutrient status, and locomotion was found to differ between the RNAi control and the CG18549 knockdown, it was decided to investigate the locomotion in more detail, Figure 6. Five- to seven-days-old male flies were collected for each cross: Driver control (daGAL4 driver), RNAi control and CG18549 knockdown (CG18549 RNAi line 1) flies and the total beam breaks were monitored and the average total beam breaks per hour was calculated. The RNAi control was once again found to be more active compared with the Driver control and the CG18549 knockdown flies while no difference between the Driver control and the CG18549 knockdown could be observed, Figure 6A. However, to further investigate the locomotory, beam breaks over time, specifying the activity of each genotype per hour, were analyzed. Overall, the RNAi control had a higher activity during several time-points times during the monitored time $(24 \mathrm{~h})$ compared with the Driver control and CG18549 knockdown flies, supporting the difference in total activity. However, at some time points, e.g., at 13.00-13.59, 14.00-14.59 and 08.00-08.59, CG18549 knockdown flies had altered activity compared with only the driver control flies, while at 00.00-00.59 G18549 knockdown flies were more active than both controls, Figure 6B.

\subsection{Dopamine Signal Transmission Genes and the Vesicular Monoamine Transporter in CG18549 Knockdown Flies}

This is the first time CG18549 has been shown to influence locomotion; therefore, gene expression of dopamine-related genes was measured, Figure 6C-E. To do so, the relative mRNA expression of the dopamine transporter (Dat), dopamine receptor subunits (Dop1R1, Dop1R2, Dop2R), tyrosine hydroxylase (Ple) and the vesicular monoamine transporter (Vmat) were measured via quantitative PCR and RNA sequencing in Driver controls, RNAi controls and ubiquitous CG18549 knockdown flies. We hypothesized that there could be a difference in these genes causing the alteration in locomotion. No statistical differences were measured for Dat, Dop1R1, Dop1R2, Dop2R and Ple, Figure 6C. No clear differences were observed for Vmat either, but a trend towards that expression is reduced in the knockdown flies compared with the Driver control flies was observed; however, it was not significant, Figure 6D. Meanwhile, the RNA sequencing data showed similar findings for Dat and Dop1R1 where no differences were observed between the three phenotypes. However, all the other genes investigated were found to be affected in the CG18549 knockdown flies compared to either the Driver control flies (Ple), alone or compared with both controls (Dop1R2, Dop2R and Vmat). Interestingly, the RNA sequencing data revealed that both Dop2R and Vmat were also significantly reduced compared with both controls, while no large differences were observed between the controls, Table 2. 

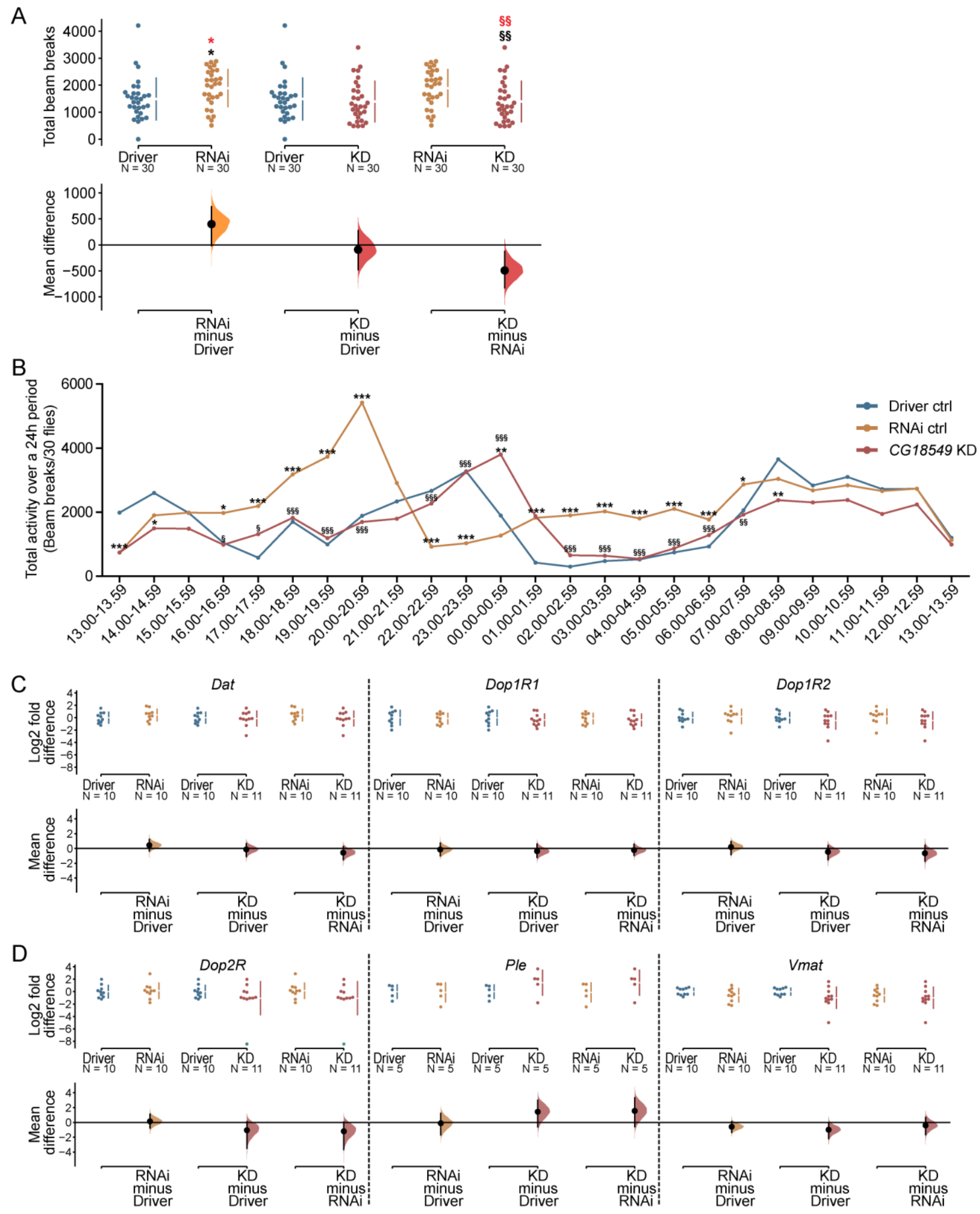

Figure 6. Cont. 


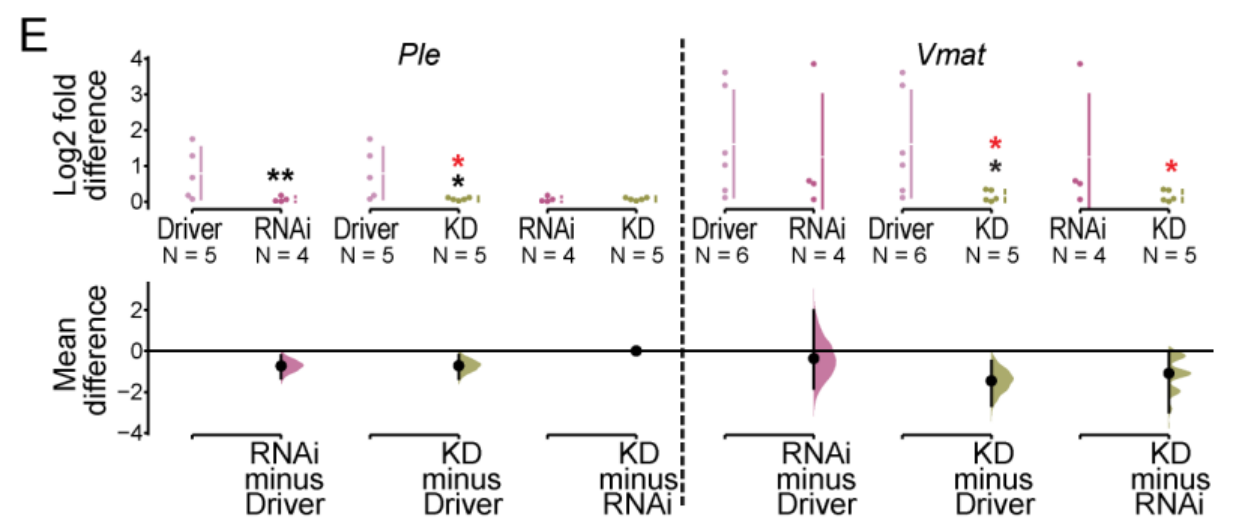

Figure 6. Ubiquitous knockdown of CG18549 and locomotion monitoring and transcriptional measurements of Dopamine signal transmission genes and the vesicular monoamine transporter in CG18549 knockdown flies. The CG18549 RNAi line 1 was used in this setup. F1 progenies from Driver control, RNAi control and CG18549 knockdown $(n=30)$ were transferred to glass tubes with enriched Jazz mix standard food and contained in the DAMS for $24 \mathrm{~h}$ to record the activity level (locomotion, beam breaks). Differences were calculated using Kruskal-Wallis with Dunn's comparison and/or MannWhitney (MW) as a post hoc test to calculate exact $p$-values ${ }^{*}$ or $\S p<0.0492,{ }^{* *}$ or $\S \S p<0.0099$, ${ }^{* * *}$ or $\S \S \S p<0.0001$ $\left({ }^{*}=\right.$ significant difference against the Driver control, $\S=$ significant difference against the RNAi control, in red effect size differences * = significant difference against the Driver control, $\S=$ significant difference against the RNAi control). (A) Mean differences for 3 comparisons are shown in the above Cumming estimation plot. The raw data is plotted on the upper axes; each mean difference is plotted on the lower axes as a bootstrap sampling distribution. Mean differences are depicted as dots; 95\% confidence intervals are indicated by the ends of the vertical error bars. Kruskal-Wallis $p=0.0112$; RNAi ctrl mins Driver ctrl: MW $p=0.0225$, effect size $4 \times 10^{2}\left[95.0 \% \mathrm{CI}-12.8,7.37 \times 10^{2}\right] p=0.04$; CG18549 knockdown minus Driver ctrl: MW $p=0.3061$, effect size $-91.4\left[95.0 \% \mathrm{CI}-4.76 \times 10^{2}, 2.78 \times 10^{2}\right] p=0.654$; CG18549 knockdown minus RNAi ctrl: MW $p=0.0075$, effect size is $\left.-4.92 \times 10^{2}\left[95.0 \% \mathrm{CI}-8.27 \times 10^{2},-1.24 \times 10^{2}\right] p=0.0124\right)$. (B) Total activity over time. (C-E) The ubiquitous da-GAL4 driver, $\mathrm{w}^{1118}$ and CG18549 RNAi line 1 and 2 were used. Gene expressions of Dat, Dop1R1, Dop1R2, Dop2R, Ple and Vmat were measured using qPCR, log2 fold changes were calculated according to the delta Ct method using three reference genes (Actin42a, Rpl11, Rp49) for CG18549 RNAi line 1 and controls, while one reference gene (Actin42a) was used for CG18549 RNAi line 2 and controls. The Driver control was set as control. Kruskal-Wallis with Dunn's comparison against the control was used to initially analyze differences, exact p-values were calculated using multiple corrected Mann-Whitney as a post hoc test ${ }^{*} p<0.0491,{ }^{* *} p<0.0099,{ }^{* * *} p<0.0001{ }^{*}=$ significant difference against the Driver control, $\S=$ significant difference against the RNAi control, in red effect size differences * $=$ significant difference against the Driver control, $\S=$ significant difference against the RNAi control). (C,D) Data of CG18549 RNAi line 1. The mean differences for 18 comparisons are shown in the above Cumming estimation plot. The raw data is plotted on the upper axes; each mean difference is plotted on the lower axes as a bootstrap sampling distribution. Mean differences are depicted as dots; 95\% confidence intervals are indicated by the ends of the vertical error bars. (C) Dat: Kruskal-Wallis $p=0.5425$, effect size unpaired mean difference RNAi ctrl minus Driver ctrl: 0.439 [95.0\% CI - 0.333, 1.21] $p=0.295 ;$ CG18549 knockdown minus Driver ctrl: -0.128 [95.0\% CI $-1.09,0.64] p=0.786$; CG18549 knockdown minus RNAi ctrl: -0.567 [95.0\% CI -1.52, 0.239] $p=0.247)$; Dop1R1: Kruskal-Wallis $p=0.7009$, effect size unpaired mean difference RNAi ctrl minus Driver ctrl: -0.142 [95.0\% CI -0.984, 0.707] $p=0.76$; CG18549 knockdown minus Driver ctrl -0.363 [95.0\% CI -1.2, 0.582] $p=0.43$; CG18549 knockdown minus RNAi ctrl: is -0.221 [95.0\% CI-0.922, 0.574] $p=0.582$ ); Dop1R2: Kruskal-Wallis $p=0.3470$, effect size unpaired mean difference RNAi ctrl minus Driver ctrl: 0.192 [95.0\% CI -0.822, 0.929] $p=0.672 ;$ CG18549 knockdown minus Driver ctrl -0.455 [95.0\% CI $-1.52,0.369] p=0.392$; CG18549 knockdown minus RNAi ctrl: -0.647 [95.0\% CI -1.7, 0.413] $p=0.269$ ). (D) Dop2R: Kruskal-Wallis $p=0.2889$, effect size unpaired mean difference RNAi ctrl minus Driver ctrl: 0.141 [95.0\% CI -0.768, 1.09] $p=0.782$; CG18549 knockdown minus Driver ctrl -1.06 [95.0\% CI - 3.5, 0.0975] $p=0.278$; CG18549 knockdown minus RNAi ctrl: -1.2 [95.0\% CI -3.65, 0.0492] $p=0.222$ ); Ple: Kruskal-Wallis $p=0.2276$, effect size unpaired mean difference RNAi ctrl minus Driver ctrl: -0.115 [95.0\% CI -1.7, 1.23] $p=0.927 ;$ CG18549 knockdown minus Driver $\operatorname{ctrl} 1.43$ [95.0\% CI -0.615, 2.98] $p=0.188$; CG18549 knockdown minus RNAi ctrl: 1.55 [95.0\% CI -0.584 , 3.33] $p=0.22)$; Vmat: Kruskal-Wallis $p=0.1780$, effect size unpaired mean difference RNAi ctrl minus Driver ctrl: -0.585 [95.0\% CI -1.35, 0.106] $p=0.145 ;$ CG18549 knockdown minus Driver ctrl -0.991 [95.0\% CI -2.19, -0.115] $p=0.0804 ;$ CG18549 knockdown minus RNAi ctrl: -0.406 [95.0\% CI -1.67, 0.658] $p=0.549$ ). (E) Data of CG18549 RNAi line 2. Cummings estimation plot with same setup as in B and C: Ple (Kruskal-Wallis $p=0.0212$; MW $p=0.0061$, effect size RNAi ctrl minus 
Driver ctrl: MW -0.725 [95.0\% CI -1.33, -0.215] $p=0.0568 ;$ CG18549 knockdown minus Driver ctrl: MW $p=0.0101$, effect size -0.716 [95.0\% CI -1.35, -0.2] $p=0.0252$; CG18549 knockdown minus RNAi ctrl: MW $p=1$, effect size 0.00867 [95.0\% CI $-0.0823,0.0645] p=0.883$ ); Vmat (RNAi ctrl minus Driver ctrl: MW $p=0.0823$, effect size -0.362 [95.0\% CI $-1.84,1.98]$ $p=0.687$; CG18549 knockdown minus Driver ctrl: MW $p=0.0411$, effect size $-1.45[95.0 \%$ CI $-2.68,-0.497] p=0.0394$; CG18549 knockdown minus RNAi ctrl: MW $p=0.0823$, effect size $-1.09[95.0 \%$ CI $-2.99,-0.11] p=0.035$.

To measure if the results are linked to CG18549 knockdown, one gene that was not regulated $(P l e)$ and one gene that was regulated (Vmat) were measured using the second RNAi line (CG18549 RNAi line 2). The gene expression of Ple and Vmat were measured in ubiquitous CG18549 knockdown male flies and controls aged five to seven days, Figure 6E. Ple was found to be downregulated in both the RNAi control and the CG18549 knockdown flies compared with the Driver control flies, while no difference was observed between the RNAi control and CG18549 knockdown flies, a result that is consistent with the verification result of the knockdown. A small difference in Vmat expression was detected in CG18549 knockdown flies compared with both controls Figure 6E. These results together with the locomotion behavior of ubiquitous CG18549 knockdown suggest that there are differences present, and the effect on Vmat in the first RNAi line as well as in the second RNAi line suggest that it could be linked to the vesicular monoamine transport. However, it is also likely that there are compensatory mechanisms activated in the CG18549 knockdown flies which cause the phenotype to not be prominent.

\subsection{RNA Sequencing on CG18549 Knockdown Flies and Controls}

RNA sequencing was performed on Driver control (da-GAL4, RNAi control and CG18549 knockdown (CG18549 RNAi line 1) to investigate if CG18549 knockdown affects parts of or whole cellular pathways. No obvious pathways were pinpointed and gene expression that was affected in CG18549 knockdown samples appeared in several different clusters, including non-coding RNA, miRNA, protein-coding genes and more, Supplementary Data sheet 1 and Figure S1. Instead, the RNA sequencing data, Table 2, were used to confirm the metabolism and activity related genes that were measured with qPCR in Figures 5 and 6. Similar alteration patterns were observed for CG18549 (down), Akh (up), AkhR (down), Ilp2 (down), Ilp3 (no change), Dat (no change), Dop1R2 (no change), Dop2R (down) and VMAT (down). Meanwhile Dop1R1 was found to be downregulated in the CG18549 knockdown flies compared with the Driver control flies, a trend that is also observed in the qPCR data; however, it was not confirmed by statistics. Ilp5 (qPCR: down; RNAseq: up) and Ple (qPCR: up-trend; RNAseq: down) were found to have the opposite regulation compared with the qPCR, Table 2. Interestingly, the RNA sequencing data on Ple is consistent with the qPCR data measured in the second RNAi line, Figure 6D.

\subsection{Conditional Knockdown of CG18549 in Adults Using the Elav-GAL4:GAL80 Alters Movement and Gene Expression of the Vesicular Monoamine Transporter}

If the inconsistent findings found for the ubiquitous knockdown of CG18549 are due to compensatory mechanisms, a conditional knockdown could aid in detangling the link between CG18549 and locomotion. Therefore, monitoring of locomotion behavior was performed using adult flies from the CG18549 RNAi line 1 and the conditional brain cell driver elav-GAL4:GAL80. The locomotion assay was run for $48 \mathrm{~h}$. During the first $24 \mathrm{~h}$, no significant difference was observed, Figure $7 \mathrm{~A}$, but effect size estimations suggest that CG18549 knockdown flies had altered locomotion compared with both controls. During the second day, the CG18549 knockdown flies and RNAi controls moved more than the Driver control flies, Figure 7A. In an attempt to study if the difference in locomotion was due to differences in initiating and terminating a movement, filming, and manually scoring of starts and stops, was performed for each genotype, but no differences were observed, Figure 7B. When looking at the locomotion per hour during $24 \mathrm{~h}$, it was clear that CG18549 knockdown flies were more active compared to both controls, even if the RNAi control flies were more active than the Driver control flies, as well for 16 of the $24 \mathrm{~h}$, Figure $7 \mathrm{C}$. To establish the findings in the ubiquitous knockdown flies, the gene 
expression of Dat, Dop1R1, Dop2R, Ple and Vmat were measured again but in the conditional knockdown. Vmat was found to be downregulated in both the RNAi control and CG18549 knockdown, while no alterations of Dat, Dop1R1, Dop2R and Ple were measured using qPCR, Figure 7C. These results suggest that the conditional knockdown produce progenies with a clearer phenotype compared with ubiquitous knockdown, making it possible that it is compensatory mechanisms that cause the less prominent phenotype in the ubiquitous knockdown. However, compensatory mechanisms would need to be investigated in a greater depth to conclude what are the mechanisms that compensate.

A

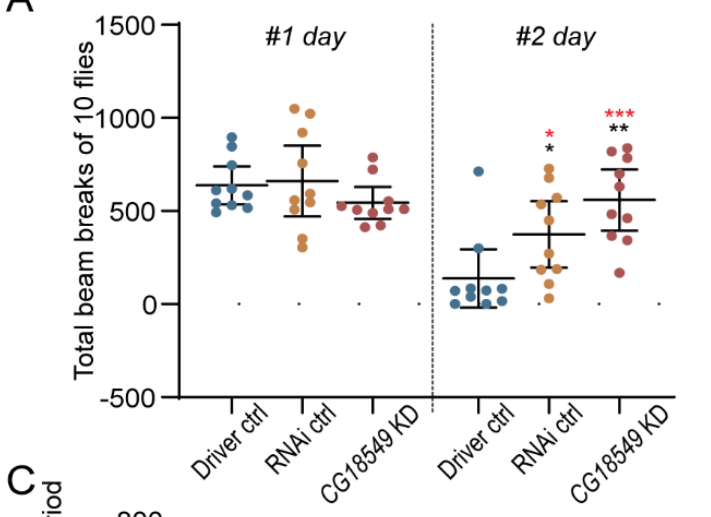

B

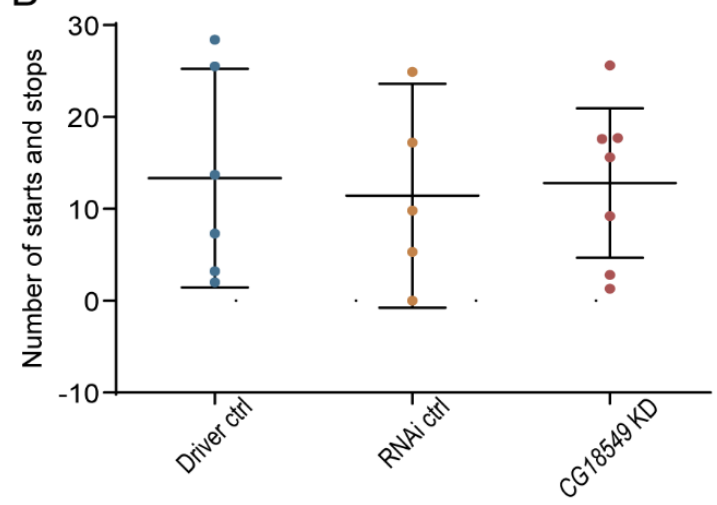

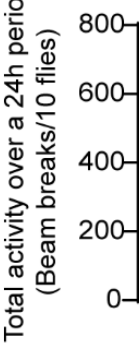

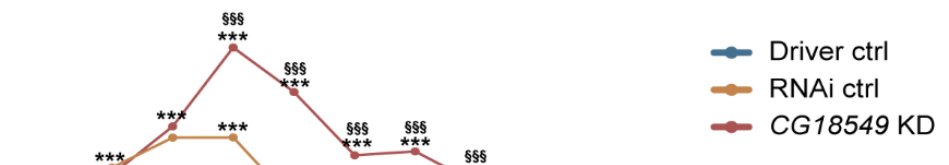

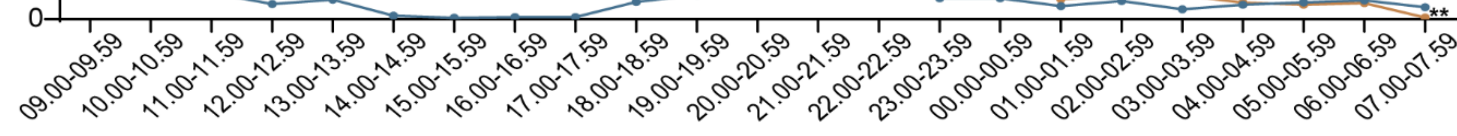

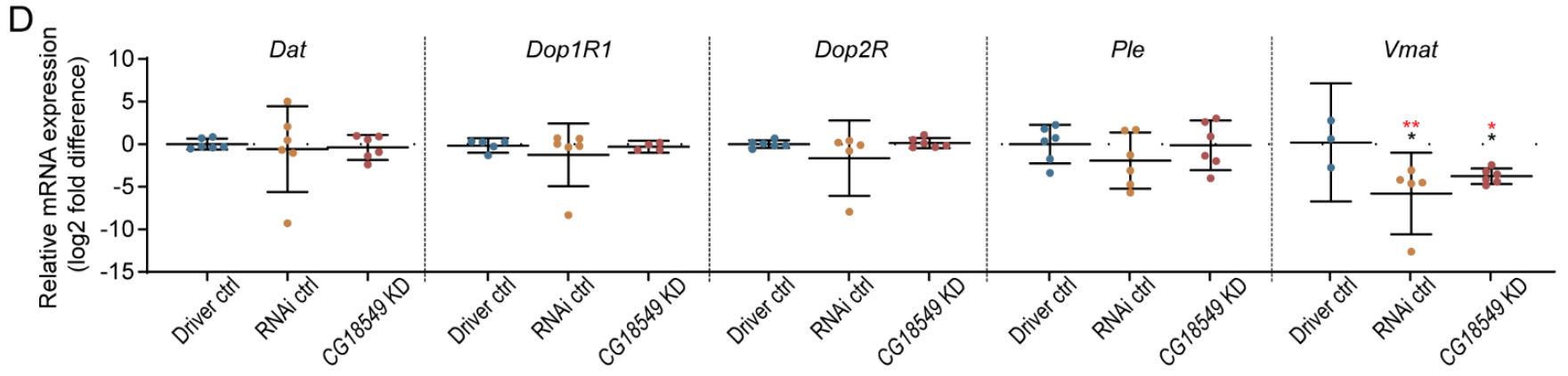

Figure 7. Locomotion of CG18549 RNAi line 1 using the elav-GAL4:GAL80 driver. Conditional (onset one day post-eclosion) brain CG18549 knockdown was performed, and activity was measured in the DAMS system. Five- to seven-days-old male flies $(n=10)$ were transferred to glass tubes prepared with standard Jazz mix food for $48 \mathrm{~h}$. Differences were calculated using Kruskal-Wallis with Dunn's comparison and/or Mann-Whitney as a post hoc test to calculate exact $p$-values ${ }^{*}$ or $\S p<0.0492$, ${ }^{* *}$ or $\S \S p<0.0099,{ }^{* * *}$ or $\S \S \S p<0.0001{ }^{*}=$ significant difference against the Driver control, $\S=$ significant difference against the RNAi control, in red effect size differences * = significant difference against the Driver control, $\S=$ significant difference against the RNAi control). (A) Scatter plot displaying the total beam breaks of run 1 (Kruskal-Wallis $p=0.1792$ ) and run 2 (Kruskal-Wallis $p=0.0023$, Mann-Whitney CG18549 knockdown ${ }^{*} p=0.0017$, RNAi $\operatorname{ctrl}{ }^{*} p=0.0211$, Mean difference for 3 comparisons were calculated with $95 \%$ confidence intervals. (in red effect size differences ${ }^{*}=$ significant difference against the Driver control, $\S=$ significant difference against the RNAi control): day \#1 unpaired mean difference RNAi ctrl minus Driver ctrl: $22.3\left[95.0 \%\right.$ CI $\left.-1.54 \times 10^{2}, 1.97 \times 10^{2}\right] p=0.811 ; C G 18549$ knockdown minus Driver ctrl: $-94.7\left[95.0 \% \mathrm{CI}-2.02 \times 10^{2}, 14.8\right] p=0.127$; CG18549 knockdown minus RNAi ctrl: $-1.17 \times 10^{2}\left[95.0 \% \mathrm{CI}-2.97 \times 10^{2}, 52.8\right]$ $p=0.225)$, day \#2 unpaired mean difference RNAi ctrl minus Driver ctrl: $2.36 \times 10^{2}\left[95.0 \%\right.$ CI $\left.4.7,3.98 \times 10^{2}\right] p=0.039$; 
CG18549 knockdown minus Driver ctrl: $4.21 \times 10^{2}\left[95.0 \%\right.$ CI $\left.1.89 \times 10^{2}, 5.71 \times 10^{2}\right] p=0.001$; CG18549 knockdown minus RNAi ctrl: $1.85 \times 10^{2}\left[95.0 \% \mathrm{CI}-18.6,3.79 \times 10^{2}\right] p=0.0908$. (B) Number of starts and stops for each genotype. (C) Total activity plotted over time $\left({ }^{*}=\right.$ statistical difference against Driver control, $\S=$ statistical difference against RNAi control, ${ }^{*} p<0.05$, $\left.{ }^{* *} p<0.001,{ }^{* * *} p<0.0001\right)$. (D) Gene expressions of Dat, Dop1R1, Dop2R, Ple and Vmat were measured using qPCR, log2 fold changes were calculated according to the delta Ct method using one reference genes (Actin42a), the Driver control was set as control. Kruskal-Wallis with Dunn's comparison against the control was used to initially analyze differences, exact $p$-values were calculated using multiple corrected Mann-Whitney as a post hoc test ${ }^{*} p<0.0491,{ }^{* *} p<0.0099$, ${ }^{* * *} p<0.0001{ }^{*}=\operatorname{significant}$ difference against the Driver control, $\S=$ significant difference against the RNAi control. No differences were measured for Dat, Dop1R1, Dop2R and Ple, while Vmat was found to be downregulated in the RNAi ctrl (Mann-Whitney * $p=0.0357$ ) and CG18549 knockdown (Mann-Whitney ${ }^{*} p=0.0476$ ) flies. Dat (Kruskal-Wallis $p=0.9107$, unpaired mean difference RNAi ctrl minus Driver ctrl: -0.591 [95.0\% CI $-5.12,2.22] p=0.84$; CG18549 knockdown minus Driver ctrl: is -0.387 [95.0\% CI $-1.57,0.631]$ $p=0.547$; CG18549 knockdown minus RNAi ctrl: 0.205 [95.0\% CI -2.63, 4.82] $p=0.938$ ), Dop1R1 (Kruskal-Wallis $p=0.6546$, unpaired mean difference RNAi ctrl minus Driver ctrl: is -1.1 [95.0\% CI -5.33, 0.647] $p=0.86$; CG18549 knockdown minus Driver ctrl: is -0.148 [95.0\% CI $-0.683,0.644] p=0.741$; CG18549 knockdown minus RNAi ctrl: 0.948 [95.0\% CI -0.771, 4.06] $p=0.945$ ), Dop2R (Kruskal-Wallis $p=0.7604$, unpaired mean difference RNAi ctrl minus Driver ctrl: -1.66 [95.0\% CI -6.45, 0.109] $p=0.234$; CG18549 knockdown minus Driver ctrl: 0.125 [95.0\% CI -0.346, 0.686] $p=0.676$; CG18549 knockdown minus RNAi ctrl: 1.78 [95.0\% CI $-0.0278,6.58] p=0.178$ ), Ple (Kruskal-Wallis $p=0.4785$, unpaired mean difference RNAi ctrl minus Driver ctrl: -1.93 [95.0\% CI $-4.51,1.13] p=0.228$; CG18549 knockdown minus Driver ctrl: is -0.14 [95.0\% CI $-2.69,2.43]$ $p=0.941$; CG18549 knockdown minus RNAi ctrl: 1.79 [95.0\% CI -1.25, 4.86] $p=0.311$ ) and Vmat (Kruskal-Wallis * $p=0.0351$; Driver ctrl vs RNAi ctrl MW * $p=0.0357$, Driver ctrl vs. CG18549 knockdown MW* $p=0.0476$; unpaired mean difference RNAi ctrl minus Driver ctrl: -6.01 [95.0\% CI -10.5, -2.67] $p=0.005$; CG18549 knockdown minus Driver ctrl: -3.97 [95.0\% CI -6.27 , $-0.931] p=0.0188$; CG18549 knockdown minus RNAi ctrl: 2.04 [95.0\% CI -0.0492, 7.1] $p=0.188$ ).

\subsection{Brain Knockdown of CG18549 Alters Locomotion in Five- to Seven-Days-Old Male Flies}

CG18549 has now been revealed to affect locomotion when using a conditional driver line specific for the brain in D. melanogaster. Despite conditional knockdown being a good model to study gene knockdown during latter stages of life, the GAL4:GAL80 system requires that the crosses are maintained at a higher temperature to dissolve the GAL80 from the GAL4 to activate the transcription. Higher temperature can, on its own, affect the fly; therefore, subsequently, we wanted to study the effects of embryonic brain knockdown of CG18549 using the elav-GAL4 driver to study if the phenotype remained detectable.

The flies from the CG18549 knockdown and control crosses were used in a total of three locomotory runs using the DAMS, Figure 8A. CG18549 knockdown flies were found to move more compared with the Driver control in all three runs, but CG18549 knockdown flies were only found to move more than the RNAi control during the second run; moreover, the RNAi control moved more than the Driver control. A representative plot of total activity over time of ten flies were drawn to illustrate the difference in locomotion per hour, Figure 8B. Here it was possible to observe a difference in activity during specific timepoints, and CG18549 knockdown flies were monitored to move more at 19.00-19.59 and 09.00-09.59 compared with both controls, while at several timepoints knockdown flies moved more than the driver control.

\subsection{Brain Knockdown of CG18549 and Locomotion in Elderly Flies}

Age has been shown to have an effect on locomotion responses and changes in monoamine levels and metabolites in mice [38]. In fly, Vmat expression has been found to be of great importance to locomotive behavior [39]. Therefore, we wanted to study how age affects locomotion in CG18549 knockdown flies that also display an already existing reduction in Vmat expression. An age-dependent locomotion assay was performed on flies (elav-GAL4 driver and CG18549 RNAi line 1) aged 13 and 21 days. RNAi controls and CG18549 knockdowns were found to be less active at an age of 13 days old compared with the Driver control, Figure $8 \mathrm{C}$, while no differences in activity could be measured in 21-days-old flies, Figure 8D. When plotting activity over time for flies aged to 13 days, the Driver control was found to have twice as high activity, Figure 8E, while at an age of 21 days, all three, the Driver control, the RNAi control and CG18549 knockdown flies moved 
equally, Figure 8F. However, the assay for the flies aged to 13 days contain fewer flies and the variation is larger within the groups, hence this particular result should be interpreted with caution. Taken together, the results suggest that CG18549 knockdown and RNAi control flies have an earlier onset of slower movement compared with Driver control flies.
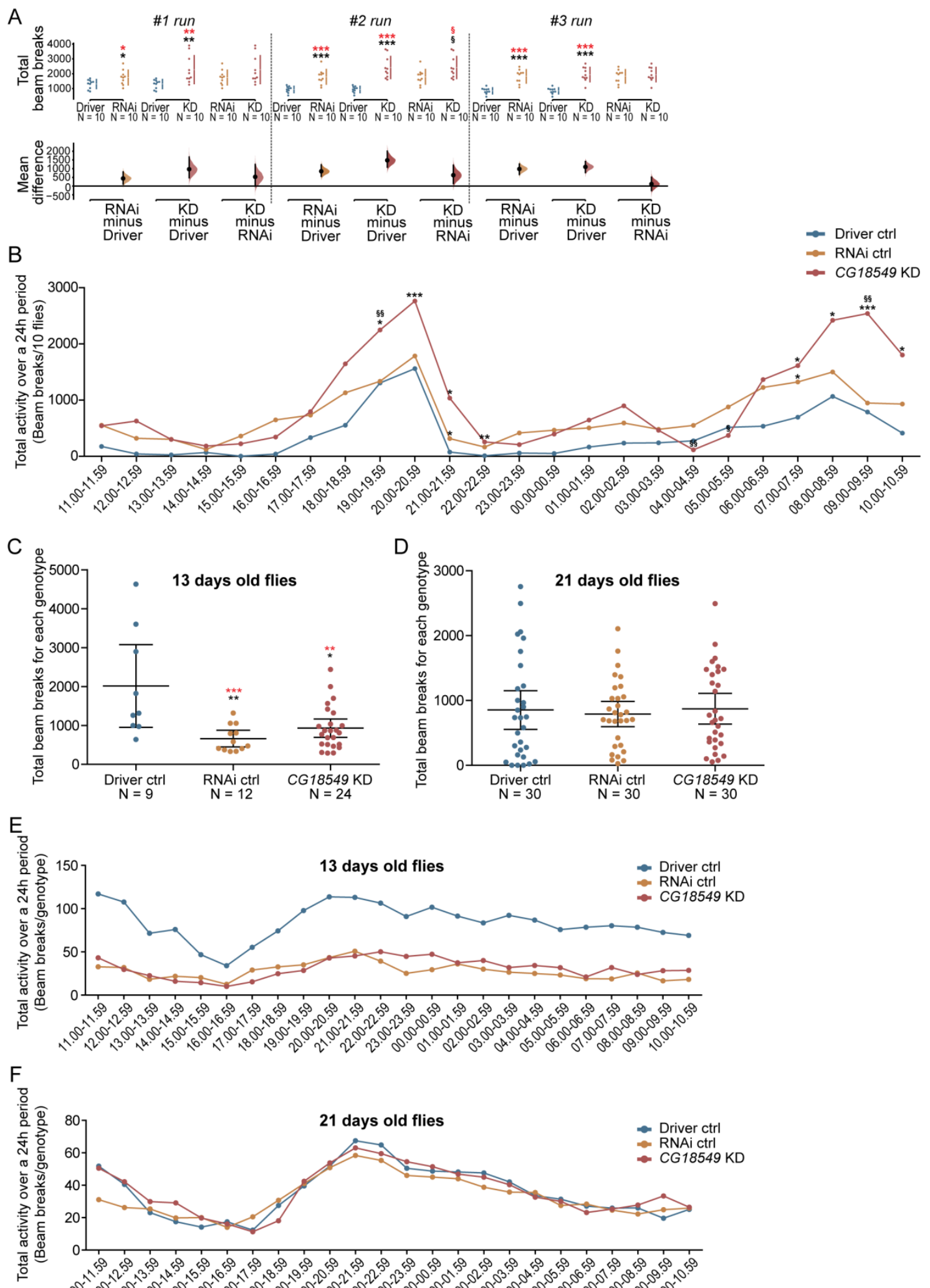

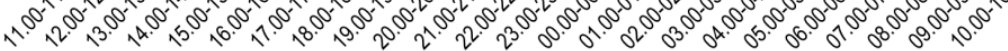


Figure 8. Locomotion on CG18549 RNAi line 1 using the elav-GAL4 driver. Brain CG18549 knockdown was performed, and activity was measured in the DAMS system. Five- to seven-days-old male flies $(n=10)$ were transferred to glass tubes prepared with standard Jazz mix food for $72 \mathrm{~h}$. Differences were calculated using Kruskal-Wallis with Dunn's comparison and/or Mann-Whitney as a post hoc test to calculate exact $p$-values ${ }^{*}$ or $\S p<0.0492,{ }^{* *}$ or $\S \S p<0.0099,{ }^{* * *}$ or $\S \S \S p<0.0001{ }^{*}=$ significant difference against the Driver control, $\S=$ significant difference against the RNAi control, in red effect size differences $*$ significant difference against the Driver control, $\S=$ significant difference against the RNAi control). (A) Mean differences for 9 comparisons are shown in the above Cumming estimation plot. The raw data is plotted on the upper axes; each mean difference is plotted on the lower axes as a bootstrap sampling distribution. Mean differences are depicted as dots; $95 \%$ confidence intervals are indicated by the ends of the vertical error bars. Kruskal-Wallis Run $1 p=0.0093$, Run $2 p \leq 0.0001$, Run $3 p \leq 0.0001$, Driver ctrl and RNAi Run $1: 4.39 \times 10^{2}$ $\left[95.0 \%\right.$ CI $\left.1.14 \times 10^{2}, 8.14 \times 10^{2}\right] p=0.0222$, Run 2: $8.52 \times 10^{2}\left[95.0 \%\right.$ CI $\left.5.58 \times 10^{2}, 1.22 \times 10^{3}\right] p=0.0$, Run 3: $9.81 \times 10^{2}[95.0 \%$ CI $\left.6.67 \times 10^{2}, 1.27 \times 10^{3}\right] p=0.0 ;$ CG18549 knockdown and Driver ctrl Run 1: $9.69 \times 10^{2}\left[95.0 \%\right.$ CI $\left.4.81 \times 10^{2}, 1.67 \times 10^{3}\right] p=0.002$, Run 2: $1.48 \times 10^{3}\left[95.0 \%\right.$ CI $\left.1.08 \times 10^{3}, 2.01 \times 10^{3}\right] p=0.0$, Run 3: $1.1 \times 10^{3}\left[95.0 \%\right.$ CI $\left.7.78 \times 10^{2}, 1.41 \times 10^{3}\right] p=0.0$; CG18549 knockdown and RNAi ctrl Run 1: $5.3 \times 10^{2}\left[95.0 \%\right.$ CI $\left.-12.5,1.25 \times 10^{3}\right] p=0.131$, Run 2: $6.28 \times 10^{2}\left[95.0 \%\right.$ CI $\left.1.47 \times 10^{2}, 1.2 \times 10^{3}\right] p=0.035$, Run 3: $1.19 \times 10^{2}\left[95.0 \% \mathrm{CI}-2.57 \times 10^{2}, 5.1 \times 10^{2}\right] p=0.579$. Mann-Whitney post hoc test Run 1: CG18549 knockdown $p=0.0029, \mathrm{RNAi}$ $\operatorname{ctrl} p=0.0355 ;$ Run 2; CG18549 knockdown * $p \leq 0.0001 \S p=0.0312$, RNAi $\operatorname{ctrl}{ }^{*} p=0.0006$; Run 3: CG18549 knockdown * $p \leq 0.0001$, RNAi $\operatorname{ctrl}^{*} p \leq 0.0001$. (B) Total activity plotted over time, ${ }^{*} p<0.05,{ }^{* *} p<0.001,{ }^{* * *} p<0.001\left({ }^{*}=\right.$ significant difference against the Driver control, $\S=$ significant difference against the RNAi control. (C-F) Brain CG18549 knockdown was performed, and activity was measured in the DAMS system. Male flies were aged to 13 (Driver $\operatorname{ctrl} n=9$, RNAi ctrl n = 12, CG18549 knockdown $n=24$ ) and $21(\mathrm{n}=30)$ days. They were transferred to glass tubes prepared with standard Jazz mix food for $72 \mathrm{~h}$. Differences were calculated using Kruskal-Wallis with Dunn's comparison and/or Mann-Whitney as a post hoc test to calculate exact $p$-values ${ }^{*} p<0.0492,{ }^{* *} p<0.0099$, ${ }^{* * *} p<0.0001\left(^{*}=\right.$ significant difference against the Driver control, $\S=$ significant difference against the RNAi control. (C,D) Scatter plot displaying the total beam breaks, (C) 13 days old flies (Kruskal-Wallis $p=0.0063$, Mann-Whitney CG18549 knockdown * $p=0.0129$, RNAi $\operatorname{ctrl}^{*} p=0.0040$; unpaired mean difference between Driver ctrl and RNAi ctrl is $-1.35 \times 10^{3}\left[95.0 \% \mathrm{CI}-2.43 \times 10^{3},-6.14 \times 10^{2}\right]$ $p=0.0008$, Driver ctrl and CG18549 knockdown is $-1.08 \times 10^{3}\left[95.0 \%\right.$ CI $\left.-2.23 \times 10^{3},-3.91 \times 10^{2}\right] p=0.0052$, RNAi ctrl and CG18549 knockdown is $2.69 \times 10^{2}\left[95.0 \% \mathrm{CI}-20.0,5.5 \times 10^{2}\right] p=0.131$ ) and (D) 21 days old flies (no differences). (E,F) Total activity plotted over time, (E) 13 days old flies and (F) 21 days old flies.

\subsection{Brain Knockdown of CG18549 Affects Stress Response in Flies}

The inconsistency in movement observed, as well as the alterations in locomotion early in the experimental setups could indicate that a stress-related phenotype is present. Also, earlier findings about the locomotion phenotype, monoamine loading and Vmat expression formed the hypothesis that the locomotion alterations observed for CG18549 flies are due to the low expression of Vmat, suggesting that the knockdown flies cannot load vesicles with enough monoamines during more active conditions such as stress. Furthermore, altered Vmat expression could cause other phenotypes such as anxiety [40]. Therefore, a stress assay, where the flies are exposed to bright light three times a day (time point indicated by arrows) for two days were performed on brain knockdown flies and controls, Figure 9. Flies were exposed to light at 09.00, 12.00 and 15.00 the first day and 09.00, 12.00 and 16.00 the second day. Initially, no differences were observed when plotting the total activity over time, but after the flies were stimulated with bright light, an altered number of beam breaks could be observed for the CG18549 knockdown flies compared with both controls, Figure 9A. Interestingly, after the third stimulation with bright light, all three genotypes recovered after an hour, but three to four hours post-stimulation, the CG18549 knockdown flies experienced a sudden drop in locomotion, which were not observed for either controls, Figure 9A, time point 18.00-18.59 until 03.00-03.59. This was observed during both days. Moreover, an increased locomotion peak was observed for the CG18549 knockdown flies after 21.00, while the controls activity decreased as normal when following the circadian rhythm. The peak was also observed during the second day, suggesting that the CG18549 knockdown flies are indeed not able to handle the bright light as the controls. In contrast, when analyzing the total beam breaks during both days, a trend of increased activity could be observed for both the RNAi control and the CG18549 knockdown flies, but they were not statistically significant, Figure $9 \mathrm{~B}$. Moreover, to study immediate stress responses the differences in activity the hour after stimulation with bright light were analyzed. Here, an 
increase in activity could be observed for the CG18549 knockdown flies, but it was only statistically significant at timepoint $13.00-13.59$ of both days, Figure 9 C, and the response to the bright light minimized as expected with each stimulation, which is observed on the total beam breaks at each time point. Taken together, the results suggest that CG18549 knockdown flies change their locomotion behavior post-stimulation and had difficulties to recover several hours after the stressor. However, there is not a clear indication that CG18549 is linked to stress-behavior and anxiety-related behavior, and further experiments are needed to establish that connection.

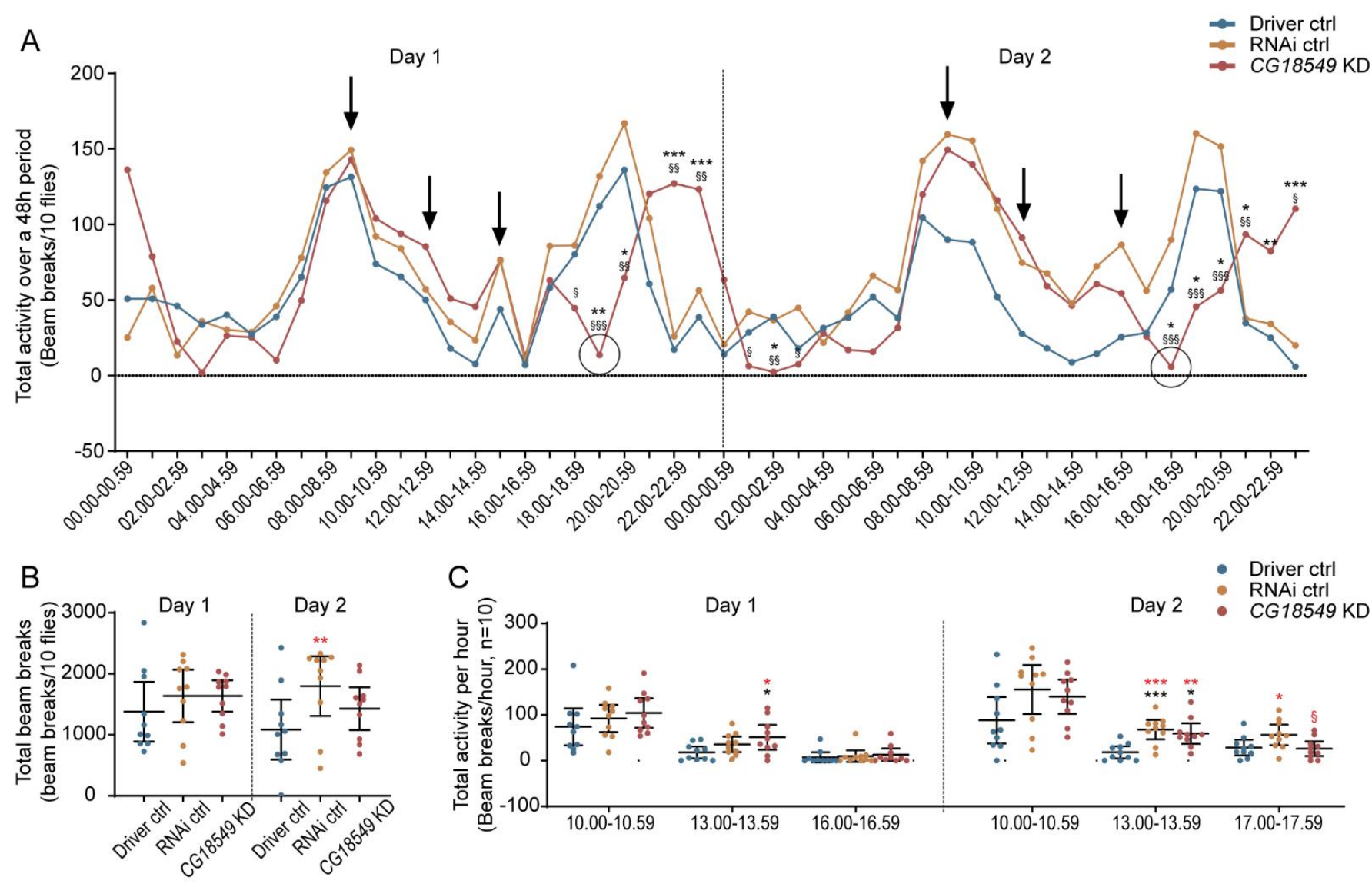

Figure 9. Brain knockdown of CG18549 affects the stress response after stimulation with bright light. Brain CG18549 knockdown using the elav-GAL4 driver and the CG18549 RNAi line 1 was performed, and flies ( $\mathrm{n}=10)$ were placed in a DAMS for $48 \mathrm{~h}$ ). Stress was induced by exposing the flies to bright light (LED, 1500 lumens) for 10 min three times $(9.00,12.00$ and 15.00/16.00) per day, Differences were calculated using Kruskal-Wallis with Dunn's comparison and/or Mann-Whitney as a post hoc test to calculate exact $p$-values ${ }^{*}$ or $\S p<0.0492,{ }^{* *}$ or $\S \S p<0.0099,{ }^{* * *}$ or $\S \S \S p<0.0001$ $\left({ }^{*}=\right.$ significant difference against the Driver control, $\S=$ significant difference against the RNAi control, in red effect size differences * = significant difference against the Driver control, $\S=$ significant difference against the RNAi control). (A) Total activity over the $48 \mathrm{~h}$ period. Black arrows illustrate the stimulation, and the two circulated points show the large dip in activity that the CG18549 knockdown flies experienced post-stimulation. (B) Total beam breaks per genotype divided into day 1 (Kruskal-Wallis $p=0.3682$, unpaired mean difference between Driver ctrl and RNA ctrl $2.56 \times 10^{2}$ [95.0\% CI $\left.-3.51 \times 10^{2}, 7.15 \times 10^{2}\right] p=0.379$, Driver ctrl and CG18549 knockdown $2.57 \times 10^{2}\left[95.0 \%\right.$ CI $\left.-2.8 \times 10^{2}, 6.53 \times 10^{2}\right]$ $p=0.301$, RNAi ctrl and CG18549 knockdown $0.8\left[95.0 \% \mathrm{CI}-3.76 \times 10^{2}, 4.42 \times 10^{2}\right] p=0.997$ ) and day 2 (Kruskal-Wallis $p=0.0599$, unpaired mean difference between Driver ctrl and RNA ctrl $7.11 \times 10^{2}\left[95.0 \%\right.$ CI $\left.69.2,1.22 \times 10^{3}\right] p=0.0384$, Driver ctrl and CG18549 knockdown $3.43 \times 10^{2}\left[95.0 \%\right.$ CI $\left.-1.68 \times 10^{2}, 8.17 \times 10^{2}\right] p=0.202$, RNAi ctrl and CG18549 knockdown $\left.-3.68 \times 10^{2}\left[95.0 \% \mathrm{CI}-8 \times 10^{2}, 1.93 \times 10^{2}\right] p=0.169\right)$. (C) Total activity one-hour post-stimulation [10.00-10.59 day 1: Kruskal-Wallis $p=0.2975$, effect size RNAi ctrl minus Driver ctrl: 18.3 [95.0\% CI -31.5, 53.7] $p=0.427$, CG18549 knockdown minus Driver ctrl: 30.1 [95.0\% CI -17.7, 70.2] $p=0.203$, CG18549 knockdown minus RNAi ctrl: 11.8 [95.0\% CI -21.0, 51.2] $p=0.548$; day 2: Kruskal-Wallis $p=0.0849$, effect size RNAi ctrl minus Driver ctrl: 67.3 [95.0\% CI -0.9 , 
$\left.1.22 \times 10^{2}\right] p=0.0576$, CG18549 knockdown minus Driver ctrl: 51.5 [95.0\% CI -4.3, 99.3] $p=0.077$, CG18549 knockdown minus RNAi ctrl: is -15.8 [95.0\% CI -66.2, 41.1] $p=0.578 ; 13.00-13.59$ day 1: Kruskal-Wallis $p=0.0664$, Mann-Whitney ${ }^{*} p=0.0444$, effect size RNAi ctrl minus Driver ctrl: 17.6 [95.0\% CI 0.7, 35.5] $p=0.0766$, CG18549 knockdown minus Driver ctrl: 33.1 [95.0\% CI 9.9, 59.9]. $p=0.0246$, CG18549 knockdown mins RNAi ctrl: 15.5 [95.0\% CI -9.6, 43.0] $p=0.285$; day 2: Kruskal-Wallis $p=0.0012,{ }^{*} p=0.0009$ and 0.0136, effect size RNAi ctrl minus Driver ctrl: 49.6 [95.0\% CI 27.7, 68.0] $p=0.0004$, CG18549 knockdown minus Driver ctrl: 41.2 [95.0\% CI 21.9, 65.0] $p=0.0012$, CG18549 knockdown minus RNAi ctrl: $-8.4[95.0 \%$ CI $-30.6,21.2] p=0.53 ; 16.00-16.59$ Kruskal-Wallis $p=0.3540$, effect size RNAi ctrl minus Driver ctrl: $2.6[95.0 \%$ CI $-10.0,18.1] p=0.64$, CG18549 knockdown minus Driver ctrl: $5.6[95.0 \%$ CI $-8.2,21.0] p=0.469$, CG18549 knockdown minus RNAi ctrl: 3.0 [95.0\% CI -12.8, 18.2] $p=0.692 ; 17.00-17.59$ Kruskal-Wallis $p=0.3682$, effect size RNAi ctrl minus Driver ctrl: 27.8 [95.0\% CI 4.0,50.6] $p=0.0414$, CG18549 knockdown minus Driver ctrl: -2.4 [95.0\% CI -22.7, 15.8] $p=0.808$, CG18549 knockdown minus RNAi ctrl: -30.2 [95.0\% CI -51.8, -6.9] $p=0.0244]$.

\section{Discussion}

MFSD11 is an evolutionary-conserved protein and related proteins are identified in both prokaryotes and eukaryotes [17,27], but still the expression and function of MFSD11 is not fully known. A couple of years ago, MFSD11 was identified as an "atypical" SLC [16], where CG18549 was suggested to be the orthologue to MFSD11. The closest relatives to MFSD11 in the human proteome are the UNC93 proteins (UNC93A and UNC93B1) [27], two proteins with very different function. UNC93A is believed to be involved in potassium fluxes [27,41,42], while UNC93B1 is established to be a transporter of toll-like receptors [43-45]. MFSD11 has also been showed to cluster with the SLC45 (proton:sugar cotransporter) and the SLC46 (folate transporter) superfamilies of the SLCs [17]. This suggests that MFSD11 could be a transporter of solubles, or it could have similar functions as UNC93A (regulatory subunit) and UNC93B1 (Receptor transporter and aid in membrane attachment). However, this still remains uncertain.

Previous data suggest that the murine $M f s d 11$ reacts to altered nutritional levels and longer periods of starvation [17] and its promoter contains several binding sites for transcriptional regulation that react to changes in general metabolism, but also to specific changes in amino acid and glucose metabolism [20]. CG18549 has been found to be altered by starvation in whole-body samples from wildtype D. melanogaster [20]; although we did not observe a clear effect in head tissue samples from $D$. melanogaster, similar differences could be observed when comparing the whole-body and the head tissue samples. It is possible that the protective environment of the brain [46] minimizes the effect starvation has on certain genes, even if the gene has several binding sites for transcription factors, enhancers and silencer that should alter its expression as a response to nutrient levels. Moreover, it could be that the transcriptional regulation is specific to certain cell types; hence, CG18549 was not affected in the head tissue because it does not execute the same function there. It is also possible that CG18549 does not have a specific role in starvation, but rather its expression is regulated by other genes. The results suggest that CG18549 is a more stable gene during starvation than other atypical SLCs such as CG4928, the orthologue to human UNC93A [27].

Transcriptional changes after flies have been subjected to diets with sugar and protein imbalance have been found previous in whole-body samples from D. melanogaster [20]. Here, we found that CG18549 was altered in head tissue obtained from wildtype flies fed diets with strong sugar and protein imbalances. Since CG18549 expression was altered both during high and low sugar and/or protein, no clear link to one nutrient was observed. CG18549 was not affected by a low calorie diet (2.5:2.5 g/dL sugar:yeast), which was observed in the body tissue [20]. The mouse $M f s d 11$ has several transcription binding sites for regulation by both amino acid and glucose sensing elements [20], related bindings sites were not available in the eukaryotic promoter database [47] for D. melanogaster; hence, they were not searched for, but it is likely that CG18549 possess similar sites. If CG18549 also possess these bindings sites of different sensing elements as the human MFSD11 and mouse $M f s d 11$, it could explain why we can measure gene expression alterations in diets that are the opposite of each other, e.g., the 2.5:40 g/dL and 40:2.5 g/dL. These results 
would also then suggest that CG18549 have a more general role in the cell compared to sugar and amino acid transporters that are mainly found to be affected after a specific substrate has been altered $[20,48,49]$. However, the possibility that CG18549 is involved in solute transport remains, and it could be that CG18549 transport ions, e.g., hydrogen and sodium, that is needed of both amino acid and sugar transporters for proper function. Therefore, similar transcriptional regulation would be in place during different diets, since its role would be to maintain ion balance. However, it is still only speculation, but similar functions have been observed for its close relative Unc93a [27,32].

In addition, an effect on the adipokinetic hormone $(A k h)$ and its receptor $(A k h R)$ [50-52] in CG18549 knockdown flies were observed, which suggest that CG18549 could have a potential role in the metabolic pathway of glucagon. CG18549 is highly expressed in the fat bodies [35], which could strengthen this theory. The locomotory phenotype observed of CG18549 knockdown flies could also possibly be explained by the transcriptional changes of Akh. Akh is involved in locomotor activity stimulation [53] and a depletion of the Akh-producing cells have been reported to result in a decreased locomotor activity [53], suggesting that the increased $A k h$ expression could promote a more active phenotype.

CG18549 knockdown and RNAi controls flies were found to have an earlier onset of locomotory decline that are observed with age [54]. Interestingly, the CG18549 knockdown flies also reacted stronger to bright light stimulation compared with both controls and both locomotory activity alterations, as well as changes in the rhythm were observed, suggesting that the flies cope with that kind of stressor differently. It is therefore possible that an anxiety-related phenotype is present $[55,56]$, but more studies are needed to confirm the speculation. CG18549 was abundantly expressed in the larvae and adult CNS, but no double-immunostaining was performed; hence, certain neuronal and/or glial populations were not identified. This makes it difficult to give a detailed explanation to the locomotory phenotype. According to previous findings in mice [17], MFSD11 was found to overlap with anti-glutaminase [17]. Furthermore, MFSD11 was found in vesicular inhibitory amino acid transporter (VIAAT)-eGFP positive cells [17]. Taken together, the expression profile in mice suggest that MFSD11 is expressed in different neuronal subpopulations and the authors suggest that MFSD11 is expressed in both excitatory and inhibitory subpopulations [17].

Recently, shared behavior processes between arthropods and vertebrates were discovered, and the central complex and the basal ganglia were found to have a deep homology [57]. Dysfunctions in both these systems cause behavioral defects such as motor abnormalities, impaired memory formation and more [57]. Dysregulation in the central complex could be one explanation to why CG18549 knockdown flies have an altered locomotion. For example, Vmat expression was affected by CG18549 knockdown, and altered packaging of neurotransmitters could be a reason to the locomotion pattern. In humans, there are two VMATs (VMAT1 and VMAT2) that act as exchangers of monoamine and hydrogen, and are responsible for packaging, e.g., dopamine, serotonin, and norepinephrine in vesicles [58]. In D. melanogaster, there is one Vmat orthologue that encodes a protein responsible for packaging neurotransmitters such as dopamine, serotonin and octopamine into secretory vesicles $[39,59]$. Dopamine and serotonin are also vital for locomotion $[60,61]$ and are also closely linked to stress and anxiety [55,62]. Therefore, it is possible and more likely that the locomotory phenotype that we observe were due to lowered packaging of monoamines. Furthermore, we believe that the sudden decline in activity and the long recovery time observed for the CG18549 knockdown flies after the stressor (light) was induced, support the suggestion that vesicles are not filled with the right neurotransmitter in a normal rate; therefore, the locomotory activity curve becomes skewed.

\section{Conclusions}

To conclude, this is the first in-depth characterization focusing on the physiological role of MFSD11 performed in the model organism D. melanogaster, and the study's main findings were: (I) MFSD11 and CG18549 are predicted to have very similar secondary and tertiary structure, (II) CG18549 was found to be abundantly expressed in both third instar 
larvae CNS and the adult brain, (III) CG18549 was altered by nutrient levels, especially diets with strong sugar:protein imbalance, (IV) brain knockdown of CG18549 affect locomotion and results in more active phenotype, (V) CG18549 knockdown flies were found to react differently post-stimulus of the a stressor (bright light) and (VI) Vmat expression was reduced in the CG18549 knockdown flies. These results conclude that CG18549 is evolutionary conserved, abundantly expressed in more species than mammals, the gene expression is affected by what the flies eat and that there is a link between CG18549 expression, locomotion and Vmat. However, to establish this link, e.g., the levels of neurotransmitters need to be measured, which is something that has not been undertaken here. The exact mechanisms behind all observations are not fully understood, but our study provides new insights into the expression and function of CG18549. Clearly, these results, among others about this orphan transporter, provide a strong example as to why it is vital to fully characterize them, and through that, gain knowledge about the body during normal conditions and disease.

Supplementary Materials: The following are avalaible online at https:/ /www.mdpi.com/article/10 .3390/insects12111024/s1, Figure S1: Protein ANalysis THrough Evolutionary Relationships (PANTHER) classification of RNA sequencing data, Data sheet 1: RNA seq data 2020-12-25_supplementary data, Description 1: Supplementary material Figure S1 Appendix.

Author Contributions: Conceptualization: M.M.C., E.P., M.J.W. and R.F.; Data curation: R.F.; Analysis: M.M.C. and R.F.; Funding Acquisition: R.F.; Investigation: M.M.C., F.A.L. and E.P.; Project administration: M.M.C.; Resources: M.J.W. and R.F.; Supervision: M.J.W. and R.F.; Visualization: M.M.C. and F.A.L.; Writing-Original draft preparation: M.M.C. and R.F.; Writing-Review and editing: M.M.C. All authors have read and agreed to the published version of the manuscript.

Funding: This study was supported by the Swedish Research Council (2016-01972), the Swedish Brain Foundation (FO2018-0130), the Swedish Society for Medical Research (201507), the Novo Nordisk Foundation (34224), Åhlens Foundation (193027), Engkvists Foundation (20160614), and Magnus Bergvalls Foundation (201601754). The SNP\&SEQ Platform is also supported by the Swedish Research Council and the Knut and Alice Wallenberg Foundation.

Institutional Review Board Statement: Not applicable.

Informed Consent Statement: Not applicable.

Data Availability Statement: The datasets generated for this study can be found in the SRA (SUB8801412, https:/ / submit.ncbi.nlm.nih.gov/subs/sra/SUB8801412/overview, accessed on 16 March 2021) or within the manuscript and its Supplementary Materials.

Acknowledgments: We would like to express our greatest gratitude towards the SNP\&SEQ Technology Platform in Uppsala for performing the sequencing. The facility is part of the National Genomics Infrastructure (NGI) Sweden and Science for Life Laboratory. We would also like to thank Johanna Lundgren for optimization of RNA extraction protocol and primers.

Conflicts of Interest: The authors declare that the research was conducted in the absence of any commercial or financial relationships that could be construed as a potential conflict of interest.

\section{References}

1. Almen, M.S.; Nordstrom, K.J.; Fredriksson, R.; Schioth, H.B. Mapping the human membrane proteome: A majority of the human membrane proteins can be classified according to function and evolutionary origin. BMC Biol. 2009, 7, 50. [CrossRef]

2. Hediger, M.A.; Clemencon, B.; Burrier, R.E.; Bruford, E.A. The ABCs of membrane transporters in health and disease (SLC series): Introduction. Mol. Asp. Med. 2013, 34, 95-107. [CrossRef] [PubMed]

3. Hediger, M.A.; Romero, M.F.; Peng, J.B.; Rolfs, A.; Takanaga, H.; Bruford, E.A. The ABCs of solute carriers: Physiological, pathological and therapeutic implications of human membrane transport proteinsIntroduction. Pflug. Arch. 2004, 447, 465-468. [CrossRef] [PubMed]

4. Hoglund, P.J.; Nordstrom, K.J.; Schioth, H.B.; Fredriksson, R. The solute carrier families have a remarkably long evolutionary history with the majority of the human families present before divergence of Bilaterian species. Mol. Biol. Evol. 2011, 28, 1531-1541. [CrossRef]

5. Abdel-Motaal, H.; Meng, L.; Zhang, Z.; Abdelazez, A.H.; Shao, L.; Xu, T.; Meng, F.; Abozaed, S.; Zhang, R.; Jiang, J. An Uncharacterized Major Facilitator Superfamily Transporter From Planococcus maritimus Exhibits Dual Functions as a Na ${ }^{+}\left(\mathrm{Li}^{+}\right.$, $\left.\mathrm{K}^{+}\right) / \mathrm{H}^{+}$Antiporter and a Multidrug Efflux Pump. Front. Microbiol. 2018, 9, 1601. [CrossRef] 
6. Madej, M.G.; Dang, S.; Yan, N.; Kaback, H.R. Evolutionary mix-and-match with MFS transporters. Proc. Natl. Acad. Sci. USA 2013, 110, 5870-5874. [CrossRef] [PubMed]

7. Pao, S.S.; Paulsen, I.T.; Saier, M.H. Major facilitator superfamily. Microbiol. Mol. Biol. Rev. 1998, 62, 1-34. [CrossRef]

8. Reddy, V.S.; Shlykov, M.A.; Castillo, R.; Sun, E.I.; Saier, M.H. The major facilitator superfamily (MFS) revisited. FEBS J. 2012, 279, 2022-2035. [CrossRef] [PubMed]

9. Law, C.J.; Maloney, P.C.; Wang, D.N. Ins and outs of major facilitator superfamily antiporters. Annu. Rev. Microbiol. 2008, 62, 289-305. [CrossRef] [PubMed]

10. Maiden, M.C.; Davis, E.O.; Baldwin, S.A.; Moore, D.C.; Henderson, P.J. Mammalian and bacterial sugar transport proteins are homologous. Nature 1987, 325, 641-643. [CrossRef]

11. Saier, M.H.; Beatty, J.T.; Goffeau, A.; Harley, K.T.; Heijne, W.H.; Huang, S.C.; Jack, D.L.; Jahn, P.S.; Lew, K.; Liu, J.; et al. The major facilitator superfamily. J. Mol. Microbiol. Biotechnol. 1999, 1, 257-279. [PubMed]

12. Perland, E.; Fredriksson, R. Classification Systems of Secondary Active Transporters. Trends Pharmacol. Sci. 2017, 38, 305-315. [CrossRef] [PubMed]

13. Fan, S.J.; Goberdhan, D.C.I. PATs and SNATs: Amino Acid Sensors in Disguise. Front. Pharmacol. 2018, 9, 640. [CrossRef] [PubMed]

14. Cesar-Razquin, A.; Snijder, B.; Frappier-Brinton, T.; Isserlin, R.; Gyimesi, G.; Bai, X.; Reithmeier, R.A.; Hepworth, D.; Hediger, M.A.; Edwards, A.M.; et al. A Call for Systematic Research on Solute Carriers. Cell 2015, 162, 478-487. [PubMed]

15. Rask-Andersen, M.; Masuram, S.; Fredriksson, R.; Schioth, H.B. Solute carriers as drug targets: Current use, clinical trials and prospective. Mol. Asp. Med. 2013, 34, 702-710. [CrossRef] [PubMed]

16. Perland, E.; Bagchi, S.; Klaesson, A.; Fredriksson, R. Characteristics of 29 novel atypical solute carriers of major facilitator superfamily type: Evolutionary conservation, predicted structure and neuronal co-expression. Open Biol. 2017, 7, 170142. [CrossRef]

17. Perland, E.; Lekholm, E.; Eriksson, M.M.; Bagchi, S.; Arapi, V.; Fredriksson, R. The Putative SLC Transporters Mfsd5 and Mfsd11 Are Abundantly Expressed in the Mouse Brain and Have a Potential Role in Energy Homeostasis. PLoS ONE 2016, 11, e0156912. [CrossRef] [PubMed]

18. Sim, X.; Jensen, R.A.; Ikram, M.K.; Cotch, M.F.; Li, X.; MacGregor, S.; Xie, J.; Smith, A.V.; Boerwinkle, E.; Mitchell, P.; et al. Genetic loci for retinal arteriolar microcirculation. PLOS ONE 2013, 8, e65804.

19. Wang, H.; Yang, J.; Schneider, J.A.; De Jager, P.L.; Bennett, D.A.; Zhang, H.Y. Genome-wide interaction analysis of pathological hallmarks in Alzheimer's disease. Neurobiol. Aging. 2020, 93, 61-68. [CrossRef]

20. Ceder, M.M.; Lekholm, E.; Klaesson, A.; Tripathi, R.; Schweizer, N.; Weldai, L.; Patil, S.; Fredriksson, R. Glucose Availability Alters Gene and Protein Expression of Several Newly Classified and Putative Solute Carriers in Mice Cortex Cell Culture and D. melanogaster. Front. Cell Dev. Biol. 2020, 8, 579. [CrossRef]

21. Gao, Y.; Wu, N.; Wang, S.; Yang, X.; Wang, X.; Xu, B. Concurrent mutations associated with trastuzumab-resistance revealed by single cell sequencing. Breast Cancer Res. Treat. 2021, 187, 613-624. [CrossRef]

22. Anazi, S.; Maddirevula, S.; Salpietro, V.; Asi, Y.T.; Alsahli, S.; Alhashem, A.; Shamseldin, H.E.; AlZahrani, F.; Patel, N.; Ibrahim, N. Expanding the genetic heterogeneity of intellectual disability. Hum. Genet. 2017, 136, 1419-1429. [CrossRef] [PubMed]

23. Kelley, L.A.; Mezulis, S.; Yates, C.M.; Wass, M.N.; Sternberg, M.J. The Phyre2 web portal for protein modeling, prediction and analysis. Nat. Protoc. 2015, 10, 845-858. [CrossRef] [PubMed]

24. Ishida, H.; Asami, J.; Zhang, Z.; Nishizawa, T.; Shigematsu, H.; Ohto, U.; Shimizu, T. Cryo-EM structures of Toll-like receptors in complex with UNC93B1. Nat. Struct. Mol. Biol. 2021, 28, 173-180. [CrossRef]

25. Williams, M.J.; Wang, Y.; Klockars, A.; Monica Lind, P.; Fredriksson, R.; Schiöth, H.B. Exposure to Bisphenol A Affects Lipid Metabolism in Drosophila melanogaster. Basic Clin. Pharmacol. Toxicol. 2014, 114, 414-420. [CrossRef] [PubMed]

26. Skorupa, D.A.; Dervisefendic, A.; Zwiener, J.; Pletcher, S.D. Dietary composition specifies consumption, obesity, and lifespan in Drosophila melanogaster. Aging Cell 2008, 7, 478-490. [CrossRef]

27. Ceder, M.M.; Aggarwal, T.; Hosseini, K.; Maturi, V.; Patil, S.; Perland, E.; Williams, M.J.; Fredriksson, R. CG4928 Is Vital for Renal Function in Fruit Flies and Membrane Potential in Cells: A First In-Depth Characterization of the Putative Solute Carrier UNC93A. Front. Cell Dev. Biol. 2020, 8, 580291. [CrossRef]

28. Vandesompele, J.; De Preter, K.; Pattyn, F.; Poppe, B.; Van Roy, N.; De Paepe, A.; Speleman, F. Accurate normalization of real-time quantitative RT-PCR data by geometric averaging of multiple internal control genes. Genome Biol. 2002, 3, RESEARCH0034. [CrossRef]

29. Artero, R.D.; Terol-Alcayde, J.; Paricio, N.; Ring, J.; Bargues, M.; Torres, A.; Perez-Alonoso, M. saliva, a new Drosophila gene expressed in the embryonic salivary glands with homologues in plants and vertebrates. Mech. Dev. 1998, 75, 159-162. [CrossRef]

30. Williams, M.J.; Klockars, A.; Eriksson, A.; Voisin, S.; Dnyansagar, R.; Wiemerslage, L.; Kasagiannis, A.; Akram, M.; Ambrosi, V.; Hallqvist, E.; et al. The Drosophila ETV5 Homologue Ets96B: Molecular Link between Obesity and Bipolar Disorder. PLoS Genet. 2016, 12, e1006104. [CrossRef]

31. Ho, J.; Tumkaya, T.; Aryal, S.; Choi, H.; Claridge-Chang, A. Moving beyond P values: Data analysis with estimation graphics. Nat. Methods. 2019, 16, 565-566. [CrossRef]

32. Ceder, M.M.; Lekholm, E.; Hellsten, S.V.; Perland, E.; Fredriksson, R. The Neuronal and Peripheral Expressed Membrane-Bound UNC93A Respond to Nutrient Availability in Mice. Front. Mol. Neurosci. 2017, 10, 351. [CrossRef] [PubMed] 
33. Perland, E.; Hellsten, S.V.; Lekholm, E.; Eriksson, M.M.; Arapi, V.; Fredriksson, R. The Novel Membrane-Bound Proteins MFSD1 and MFSD3 are Putative SLC Transporters Affected by Altered Nutrient Intake. J. Mol. Neurosci. 2017, 61, 199-214. [CrossRef] [PubMed]

34. Chintapalli, V.R.; Wang, J.; Dow, J.A. Using FlyAtlas to identify better Drosophila melanogaster models of human disease. Nat. Genet. 2007, 39, 715-720. [CrossRef] [PubMed]

35. Gelbart, W.M.; Emmert, D.B. FlyBase High Throughput Expression Pattern Data. 2013. Available online: https://flybase.org/ reports/FBrf0212041.html (accessed on 21 June 2021).

36. Wilk, R.; Hu, J.; Blotsky, D.; Krause, H.M. Diverse and pervasive subcellular distributions for both coding and long noncoding RNAs. Genes Dev. 2016, 30, 594-609. [CrossRef]

37. Lécuyer, E.; Yoshida, H.; Parthasarathy, N.; Alm, C.; Babak, T.; Cerovina, T.; Hughes, T.R.; Tomancak, P.; Krause, H.M. Global Analysis of mRNA Localization Reveals a Prominent Role in Organizing Cellular Architecture and Function. Cell 2007, 131, 174-187. [CrossRef]

38. Hall, F.S.; Itokawa, K.; Schmitt, A.; Moessner, R.; Sora, I.; Lesch, K.P.; Uhl, G.R. Decreased vesicular monoamine transporter 2 (VMAT2) and dopamine transporter (DAT) function in knockout mice affects aging of dopaminergic systems. Neuropharma 2014, 76 Pt A, 146-155. [CrossRef]

39. Simon, A.F.; Daniels, R.; Romero-Calderon, R.; Grygoruk, A.; Chang, H.Y.; Najibi, R.; Shamouelian, D.; Salazar, E.; Solomon, M.; Ackerson, L.C.; et al. Drosophila vesicular monoamine transporter mutants can adapt to reduced or eliminated vesicular stores of dopamine and serotonin. Genetics 2009, 181, 525-541. [CrossRef]

40. Lin, Z.; Canales, J.J.; Bjorgvinsson, T.; Thomsen, M.; Qu, H.; Liu, Q.R.; Torres, G.E.; Caine, S.B. Monoamine transporters: Vulnerable and vital doorkeepers. Prog. Mol. Biol. Transl. Sci. 2011, 98, 1-46.

41. de la Cruz, I.P.; Levin, J.Z.; Cummins, C.; Anderson, P.; Horvitz, H.R. sup-9, sup-10, and unc-93 may encode components of a two-pore $\mathrm{K}^{+}$channel that coordinates muscle contraction in Caenorhabditis elegans. J. Neurosci. 2003, 23, 9133-9145. [CrossRef]

42. Levin, J.Z.; Horvitz, H.R. The Caenorhabditis elegans unc-93 gene encodes a putative transmembrane protein that regulates muscle contraction. J. Cell Biol. 1992, 117, 143-155. [CrossRef] [PubMed]

43. Lee, B.L.; Moon, J.E.; Shu, J.H.; Yuan, L.; Newman, Z.R.; Schekman, R.; Barton, G.M. UNC93B1 mediates differential trafficking of endosomal TLRs. eLife 2013, 2, e00291. [CrossRef] [PubMed]

44. Huh, J.W.; Shibata, T.; Hwang, M.; Kwon, E.H.; Jang, M.S.; Fukui, R.; Kanno, A.; Jung, D.J.; Jang, M.H.; Miyake, K.; et al. UNC93B1 is essential for the plasma membrane localization and signaling of Toll-like receptor 5. Proc. Natl. Acad. Sci. USA 2014, 111, 7072-7077. [CrossRef]

45. Pelka, K.; Bertheloot, D.; Reimer, E.; Phulphagar, K.; Schmidt, S.V.; Christ, A.; Stahl, R.; Watson, N.; Miyake, K.; Hacohen, N.; et al. The Chaperone UNC93B1 Regulates Toll-like Receptor Stability Independently of Endosomal TLR Transport. Immunity 2018, 48, 911-922.e7. [CrossRef]

46. Mergenthaler, P.; Lindauer, U.; Dienel, G.A.; Meisel, A. Sugar for the brain: The role of glucose in physiological and pathological brain function. Trends Neurosci. 2013, 36, 587-597. [CrossRef] [PubMed]

47. Cavin Perier, R.; Junier, T.; Bucher, P. The Eukaryotic Promoter Database EPD. Nucleic Acids Res. 1998, 26, 353-357. [CrossRef]

48. Hellsten, S.V.; Tripathi, R.; Ceder, M.M.; Fredriksson, R. Nutritional Stress Induced by Amino Acid Starvation Results in Changes for Slc38 Transporters in Immortalized Hypothalamic Neuronal Cells and Primary Cortex Cells. Front. Mol. Biosci. $2018,5,45$. [CrossRef]

49. Hellsten, S.V.; Lekholm, E.; Ahmad, T.; Fredriksson, R. The gene expression of numerous SLC transporters is altered in the immortalized hypothalamic cell line N25/2 following amino acid starvation. FEBS Open Bio. 2017, 7, 249-264. [CrossRef]

50. Ahmad, M.; He, L.; Perrimon, N. Regulation of insulin and adipokinetic hormone/glucagon production in flies. WIREs Dev. Biol. 2020, 9, e360. [CrossRef]

51. Chatterjee, N.; Perrimon, N. What fuels the fly: Energy metabolism in Drosophila and its application to the study of obesity and diabetes. Sci. Adv. 2021, 7, eabg4336. [CrossRef]

52. Bharucha, K.N.; Tarr, P.; Zipursky, S.L. A glucagon-like endocrine pathway in Drosophila modulates both lipid and carbohydrate homeostasis. J. Exp. Biol. 2008, 211, 3103-3110. [CrossRef] [PubMed]

53. Isabel, G.; Martin, J.R.; Chidami, S.; Veenstra, J.A.; Rosay, P. AKH-producing neuroendocrine cell ablation decreases trehalose and induces behavioral changes in Drosophila. Am. J. Physiol. -Regul. Integr. Comp. Physiol. 2005, 288, R531-R538. [CrossRef] [PubMed]

54. Bednářová, A.; Tomčala, A.; Mochanová, M.; Kodrík, D.; Krishnan, N. Disruption of Adipokinetic Hormone Mediated Energy Homeostasis Has Subtle Effects on Physiology, Behavior and Lipid Status During Aging in Drosophila. Front. Physiol. 2018, 9 , 949. [CrossRef] [PubMed]

55. Mohammad, F.; Aryal, S.; Ho, J.; Stewart, J.C.; Norman, N.A.; Tan, T.L.; Eisaka, A.; Claridge-Chang, A. Ancient Anxiety Pathways Influence Drosophila Defense Behaviors. Curr. Biol. 2016, 26, 981-986. [CrossRef]

56. Neckameyer, W.S.; Nieto-Romero, A.R. Response to stress in Drosophila is mediated by gender, age and stress paradigm. Stress 2015, 18, 254-266. [CrossRef]

57. Strausfeld, N.J.; Hirth, F. Deep homology of arthropod central complex and vertebrate basal ganglia. Science 2013, 340, 157-161. [CrossRef]

58. Lawal, H.O.; Krantz, D.E. SLC18: Vesicular neurotransmitter transporters for monoamines and acetylcholine. Mol. Asp. Med. 2013, 34, 360-372. [CrossRef] 
59. Chang, H.Y.; Grygoruk, A.; Brooks, E.S.; Ackerson, L.C.; Maidment, N.T.; Bainton, R.J.; Krantz, D.E. Overexpression of the Drosophila vesicular monoamine transporter increases motor activity and courtship but decreases the behavioral response to cocaine. Mol. Psychiatry 2006, 11, 99-113. [CrossRef]

60. Fuenzalida-Uribe, N.; Campusano, J.M. Unveiling the Dual Role of the Dopaminergic System on Locomotion and the Innate Value for an Aversive Olfactory Stimulus in Drosophila. Neuroscience 2018, 371, 433-444. [CrossRef]

61. Howard, C.E.; Chen, C.L.; Tabachnik, T.; Hormigo, R.; Ramdya, P.; Mann, R.S. Serotonergic Modulation of Walking in Drosophila. Curr. Biol. 2019, 29, 4218-4230.e8.

62. Neckameyer, W.S.; Weinstein, J.S. Stress affects dopaminergic signaling pathways in Drosophila melanogaster. Stress 2005, 8, 117-131. [CrossRef] [PubMed] 